\title{
Built environmental characteristics and diabetes: a systematic review and meta-analysis
}

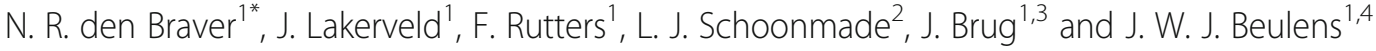

\begin{abstract}
Background: The built environment influences behaviour, like physical activity, diet and sleep, which affects the risk of type 2 diabetes mellitus (T2DM). This study systematically reviewed and meta-analysed evidence on the association between built environmental characteristics related to lifestyle behaviour and T2DM risk/prevalence, worldwide.

Methods: We systematically searched PubMed, EMBASE.com and Web of Science from their inception to 6 June 2017. Studies were included with adult populations (>18 years), T2DM or glycaemic markers as outcomes, and physical activity and/or food environment and/or residential noise as independent variables. We excluded studies of specific subsamples of the population, that focused on built environmental characteristics that directly affect the cardiovascular system, that performed prediction analyses and that do not report original research. Data appraisal and extraction were based on published reports (PROSPERO-ID: CRD42016035663).

Results: From 11,279 studies, 109 were eligible and 40 were meta-analysed. Living in an urban residence was associated with higher T2DM risk/prevalence $\left(n=19\right.$, odds ratio $\left.(\mathrm{OR})=1.40 ; 95 \% \mathrm{Cl}, 1.2-1.6 ; P^{2}=83 \%\right)$ compared to living in a rural residence. Higher neighbourhood walkability was associated with lower T2DM risk/prevalence $(n=8, \mathrm{OR}=0.79 ; 95 \% \mathrm{Cl}, 0$. $\left.7-0.9 ; P^{2}=92 \%\right)$ and more green space tended to be associated with lower T2DM risk/prevalence $(n=6, \mathrm{OR}=0.90 ; 95 \% \mathrm{Cl}$, $\left.0.8-1.0 ; P^{2}=95 \%\right)$. No convincing evidence was found of an association between food environment with T2DM risk prevalence.

Conclusions: An important strength of the study was the comprehensive overview of the literature, but our study was limited by the conclusion of mainly cross-sectional studies. In addition to other positive consequences of walkability and access to green space, these environmental characteristics may also contribute to T2DM prevention. These results may be relevant for infrastructure planning.
\end{abstract}

Keywords: Built environment, Type 2 diabetes mellitus, Lifestyle behaviour, Prevention, Urbanisation

\section{Background}

Key risk factors for type 2 diabetes mellitus (T2DM) are lack of physical activity, an unhealthy diet and lack of sleep $[1,2]$. Real-life T2DM prevention programmes aimed at changing people's lifestyle and behaviour have often been ineffective in the long term [3]. An important reason for this may be the focus on individual-level determinants of these lifestyle behaviours, such as motivation

\footnotetext{
* Correspondence: n.denbraver@vumc.nl

${ }^{1}$ Department of Epidemiology \& Biostatistics, Amsterdam Public Health

Research Institute, VU University Medical Center, De Boelelaan 1089a, 1081HV

Amsterdam, The Netherlands

Full list of author information is available at the end of the article
}

and ability, whereas they are also determined by more upstream drivers, such as the availability and accessibility of healthy options in an individual's environment. In terms of changing and sustaining healthy lifestyle behaviours, the built environment is of importance [4-7].

Urbanisation is one example of an upstream driver. Urbanisation is associated with lower total physical activity and increased consumption of processed foods, which are high in fat, added sugars, animal products and refined carbohydrates [4, 8]. However, urbanisation has also been linked to higher total walking and cycling for transportation [4]. Built environmental characteristics, 
such as higher walkability, access to parks, and access to shops and services, are consistently associated with higher physical activity [4, 5]. Food built environmental characteristics, such as the perceived availability of healthy foods, are also associated with higher diet quality. In addition, greater availability of fast-food outlets has been associated with lower fruit and vegetable consumption $[9,10]$. Other built environmental characteristics have been associated with higher stress and lack of sleep through residential noise, e.g. noise due to road and air traffic $[11,12]$.

By influencing physical activity, diet and sleep, these built environmental characteristics may also affect the risk/prevalence of T2DM. Indeed, the diabetes atlas showed higher T2DM prevalence in urban vs. rural areas [8], and a recent systematic meta-analysis reported similar results for South East Asia [13]. Two other systematic reviews addressed the association between specific built environmental characteristics and T2DM [14, 15]. However, one review only included German studies [14], while the second review included a broad range of cardiovascular disease outcomes, but only one study was included that considered T2DM as an outcome [15]. A recent meta-analysis showed that higher residential noise was associated with higher T2DM risk [16].

A comprehensive systematic review and meta-analysis of the current international evidence is, thus, lacking. This study aims to review systematically the evidence on the association between built environmental characteristics related to lifestyle behaviours and T2DM risk or prevalence, worldwide. Since characteristics of the built environment may vary with the country-specific income level, we stratified our analyses by this factor when possible. Meta-analyses were performed when three or more studies investigated the same exposure and outcome.

\section{Methods}

\section{Data sources and searches}

A literature search was performed based on the Preferred Reporting Items for Systematic Reviews and Meta-Analysis (PRISMA) statement (www.prisma-statement.org). We systematically searched the bibliographic databases PubMed, EMBASE.com and Web of Science Core Collection from their inception to 6 June 2017 (NdB and LS). Search terms included indexed terms from $\mathrm{MeSH}$ in PubMed, EMtree in EMBASE.com, as well as free-text terms. We used free-text terms only in Web of Science. Search terms expressing 'diabetes' were used in combination with search terms comprising 'environment'. Bibliographies of the identified articles were hand-searched for relevant publications. Duplicate articles were excluded. The full search strategies for all databases can be found in Additional file 1. The protocol and search strategy used were uploaded to PROSPERO prior to the study being carried out (CRD42016035663).

\section{Study selection}

Two reviewers independently screened titles, abstracts and full-text articles for eligibility $(\mathrm{NdB}$ and $\mathrm{JL}$, or JWJB). Studies were included if they: (i) studied a population of adults, 18 years or older; (ii) had T2DM incidence or prevalence, or the glycaemic markers HbA1c, glucose or insulin sensitivity as outcomes; (iii) included independent variables covering built environmental characteristics that potentially influence the risk of T2DM via lifestyle behaviours, physical activity, diet and sleep; and (iv) were written in English, Dutch or German. We excluded studies if they: (i) were not conducted in the general population, but in specific subsamples, like pregnant women, or T2DM patients; (ii) focused on built environmental characteristics that directly affect the cardiovascular system (i.e. not via lifestyle behaviours), such as exposure to particulates due to roadway proximity; (iii) performed prediction analyses or (iv) were specific publication types that do not report original scientific research (editorials, letters, legal cases and interviews). As in the general population, the vast majority of diabetes cases are T2DM (>90\%), studies were included if they did not specify the type of diabetes (type 1 diabetes mellitus or T2DM). Inconsistencies in study selection were resolved through consensus with a third reviewer (JL or JWJB).

\section{Data extraction}

One reviewer $(\mathrm{NdB})$ performed data extraction, according to a standard protocol, including measures of study design, outcome, outcome assessment and exposure assessment, demographics, and prevalence or effect measure. Data extraction was appraised by a second reviewer (JL) for a random subsample of the included studies.

\section{Quality assessment}

Two reviewers (NdB and JWJB, or JL) independently evaluated the methodological quality of the full-text papers using the Quality Assessment Tool for Quantitative Studies, as described earlier by Mackenbach et al. [17]. This tool provides a quality score based on study design, representativeness at baseline (selection bias) and follow-up (withdrawals and drop-outs), confounders, data collection, data analysis and reporting. Each domain received a weak, moderate or strong score, resulting in seven scores. A study was rated as strong when it received four strong ratings and no weak ratings. A study was rated as moderate if it received one weak rating and less than four strong ratings. Finally, a study was rated weak if it received two or more weak ratings. Study quality was assessed in terms of the reported association 
between the relevant built environmental characteristic and T2DM, even if this was not the primary analysis presented in the study. Studies with a weak rating $(n=23)$ are presented in Additional file 2 and were included in sensitivity analyses, but excluded from the main analyses.

\section{Data synthesis}

Study characteristics were described in a systematic manner, according to the built environmental characteristics under investigation. These categories were made as homogeneous as possible, based on the lifestyle behaviours. Findings were further described according to country-level income, based on the World Bank list of economies, 2016 [18].

Studies were meta-analysed when three or more studies investigated the same exposure and outcome variables. In addition, the studies had to provide at least age and sex adjusted or standardised risk ratios or prevalence, and have a moderate or strong quality rating. If reported ratios were stratified and could not be pooled with the information provided in the publication, the study's authors were contacted and asked to provide the pooled-risk ratio [19-23]. Reference categories were harmonised by taking the inverse of the risk ratio and $95 \%$ confidence interval (CI). If a risk ratio for a continuous variable was reported, we transformed this to a categorical risk ratio based on the methods of Danesh et al. [24]. Forest plots and random-effects meta-analysis models were fitted to relative risks or odds ratios. Plots and models were stratified for country income level and study quality, where permitted. In the sensitivity analyses, the studies with weak quality ratings were added to the models. Heterogeneity was tested using $I^{2}$. Analyses were performed in $\mathrm{R}$ version 3.2.5 using the Metafor package.

\section{Results}

From the 11,279 identified references, 299 full articles were screened, and 109 of these studies were included in our review, of which 23 were not included in our main analyses due to a weak quality rating (Fig. 1 and

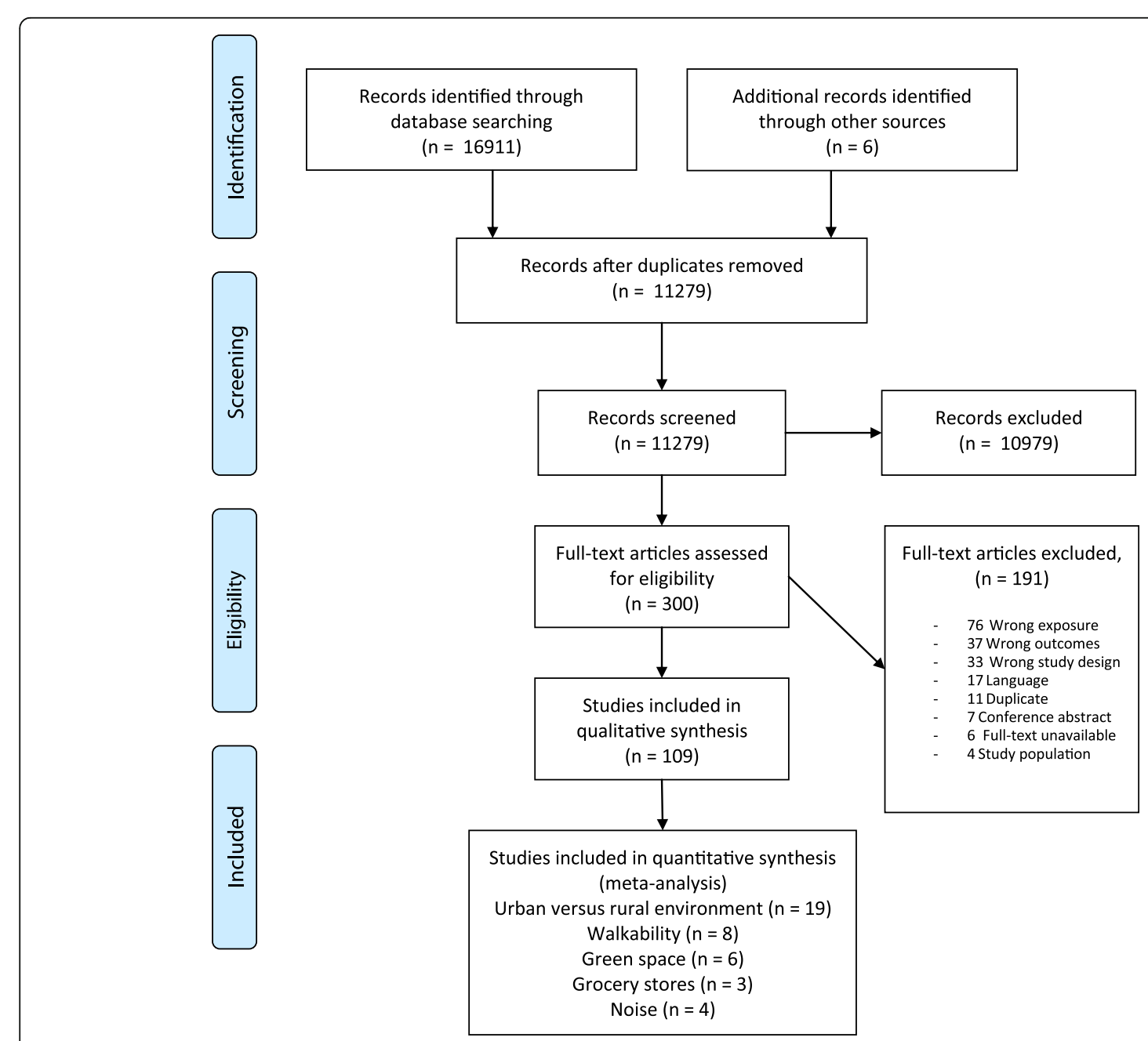

Fig. 1 Flow chart of study inclusion 
Additional file 2). Included studies were categorised according to the built environmental characteristic investigated (Tables 1 and 2), and built environments were subdivided by physical activity environment, food environment and residential noise (Table 2).

Sixty studies compared T2DM risk/prevalence in urban vs. rural environments (Table 1 and Additional file 2). The studies rated weak $(n=16)$ did not differ in terms of country income levels from the other studies [25-40].

Of the remaining 44 studies, 25 (57\%) of them found a higher risk or prevalence of T2DM in urban areas compared to rural areas. Altogether, 19 studies were eligible for the meta-analysis, which revealed a significantly higher risk/prevalence of T2DM in urban areas vs. rural areas (1.40; 95\% CI, 1.22-1.61) (Fig. 2). This association was stronger in studies with strong quality ratings (1.44; 95\% CI, 1.18-1.75), compared to those with moderate quality ratings $(1.38 ; 95 \% \mathrm{CI}, 1.11-1.70)$. After stratifying for country income level, one study was excluded [41] because the subgroup contained fewer than three studies. Associations were not different for upper-middle income countries $(1.49 ; 95 \% \mathrm{CI}, 1.16-1.92)$ and lowermiddle income countries $(1.45 ; 95 \% \mathrm{CI}, 1.20-1.74)$, but were non-significant for high-income countries (1.16; 95\% CI, 0.70-1.89).

Sensitivity analyses that included studies with weak quality ratings $[33,40]$ did not significantly change the results (Additional file 3).

Thirty studies investigated physical activity environment [19-22, 42-64] (Fig. 1, Table 2 and Additional file 2). All studies were performed in high-income level countries, except for one, which was performed in an upper-middle-level-income country [49].

Ten studies investigated the association between neighbourhood walkability and T2DM risk/prevalence. Six studies received a strong quality rating $[20,48,57$, $58,62,65]$. Six studies observed that highly walkable neighbourhoods were associated with a lower T2DM risk/prevalence [19-22, 45, 54, 65]. In the meta-analyses of six studies, a pooled-risk ratio of 0.79 (95\% CI, $0.72-$ 0.87 ) was found, with an $I^{2}$ for heterogeneity of $91.9 \%$.

Six studies investigated the association between facilities for physical activity and T2DM risk/prevalence. Three studies received a strong quality rating $[48,49$, 61]. Four studies did not observe an association between density of facilities and T2DM risk/prevalence [46, 48, $49,61]$. In two other studies, the higher availability of neighbourhood resources for physical activity was associated with lower T2DM risk $[47,63]$.

Seven studies investigated the association between green space and T2DM risk/prevalence. Two studies received a strong quality rating [44, 59]. Four studies observed that a higher availability of green space was associated with lower T2DM risk/prevalence [44, 54, 59,
64, 66]. One study observed that living closer to parks was significantly associated with higher prevalence of T2DM [64]. Aanother study observed a non-significant lower risk [42]. In meta-analyses of six studies, more green space tended to be associated with lower T2DM risk/prevalence with a pooled-risk ratio of 0.90 (95\% CI, $0.79-1.03)$ with $I^{2}$ for heterogeneity of $95.1 \%$.

Four studies investigated infrastructure in relation to T2DM risk/prevalence. Two studies received a strong quality rating $[49,67]$. Four studies did not observe an association between connectivity, infrastructure and road quality and T2DM risk/prevalence $[49,56,68]$. One study observed that a better transportation infrastructure, defined as more paved roads, was associated with higher T2DM prevalence [67].

Four studies investigated the association between safety and T2DM risk/prevalence. One study received a strong rating [49]. None of the studies showed an association between either traffic safety or safety from crime and T2DM risk/prevalence $[49,50,56]$.

Twenty studies investigated characteristics of the food environment [46-48, 51-55, 60, 61, 63, 69-77] (Fig. 1, Table 2 and Additional file 2). All studies were performed in high-income-level countries.

Eight studies investigated the association between supermarkets and grocery stores and T2DM risk/prevalence. Two studies received a strong quality rating [61, 69]. One study observed that greater availability of grocery stores was associated with lower T2DM prevalence and that a higher percentage of households without a car located far from a supermarket was associated with higher T2DM prevalence [46]. A second study observed an unadjusted correlation between a greater distance to markets and lower fasting glucose levels [53]. Five studies did not observe a significant association between availability of supermarkets/grocery stores and T2DM prevalence [60,61, 63, 69, 71, 75]. In a meta-analysis of three studies [48, 60,61], a higher density of grocery stores was not associated with T2DM risk/prevalence (1.01; 95\% CI, 0.98-1.05; $I^{2}=0 \%$ ).

Seven studies investigated the association between availability of fast-food outlets and convenience stores and T2DM risk/prevalence. Three studies received a strong quality rating $[61,69,72]$. Four studies did not observe an association between availability of fast-food outlets/convenience stores and T2DM prevalence [61, $63,69,71,75]$. A higher availability of fast-food outlets and convenience stores was associated with higher T2DM prevalence in two studies [46, 72]. Studies could not be meta-analysed because the studies did not investigate consistent outcomes (T2DM risk vs. markers).

Four studies investigated the healthiness of the food environment subjectively or as an index and the association with T2DM risk/prevalence. One study received a 


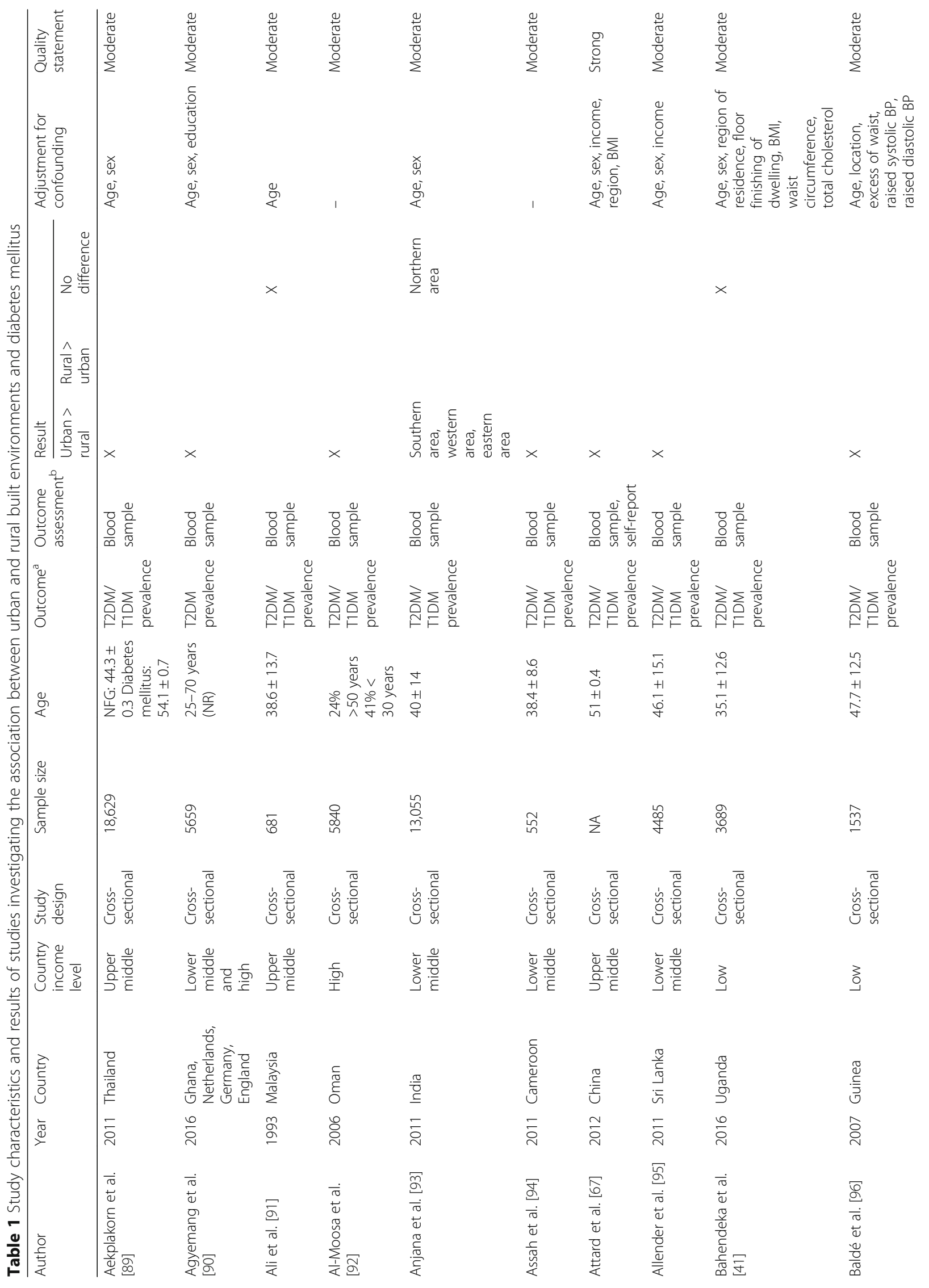




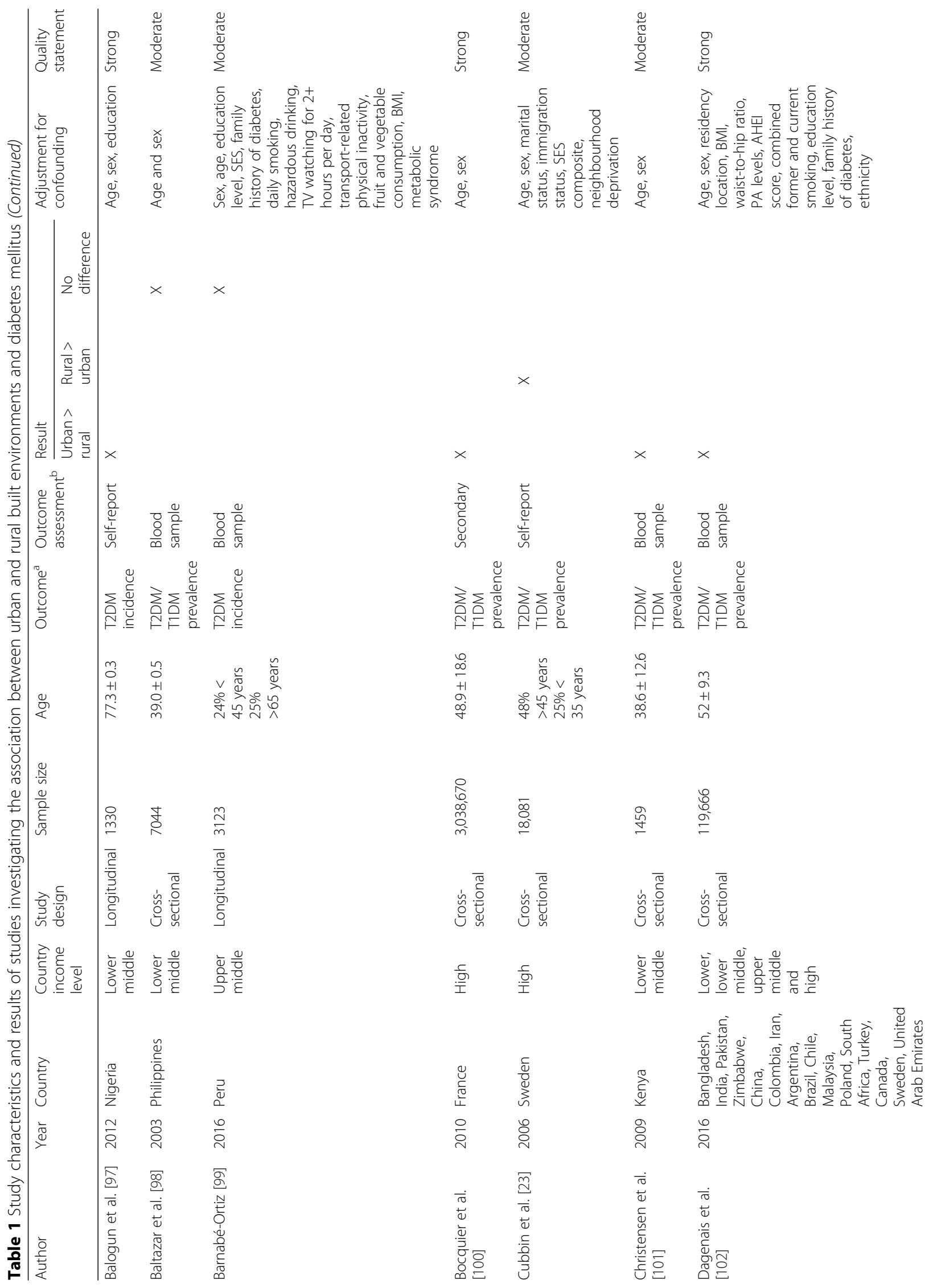




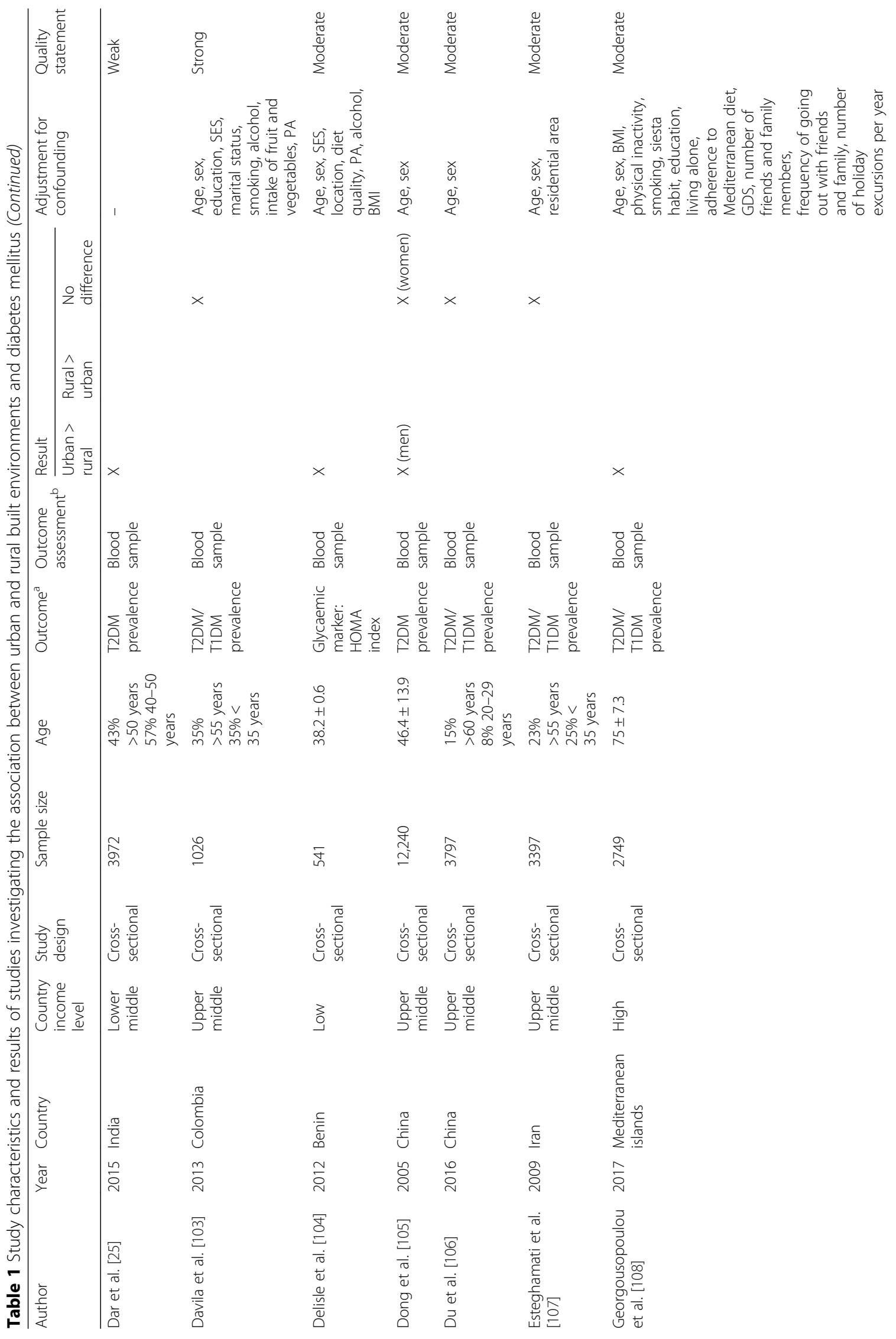




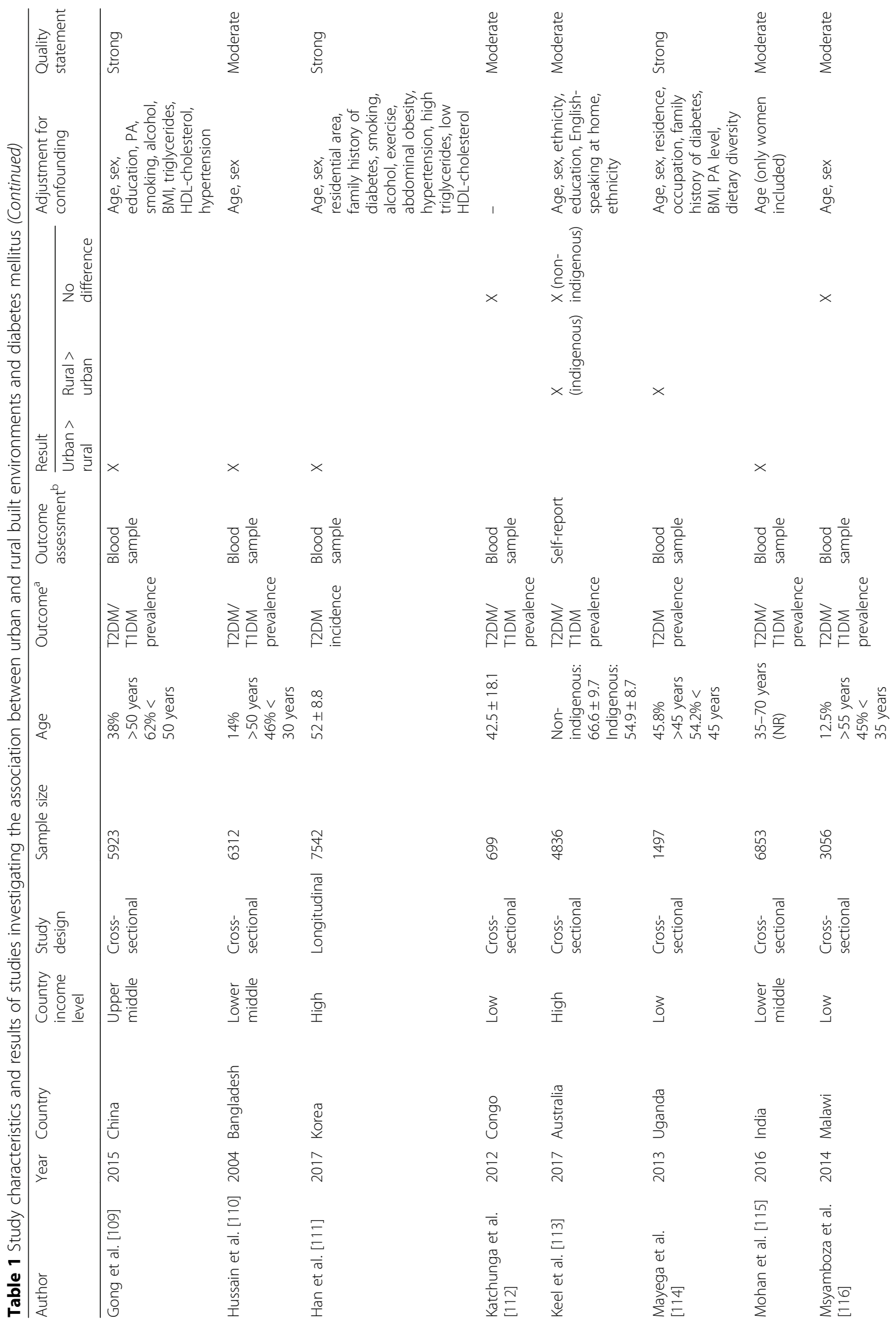




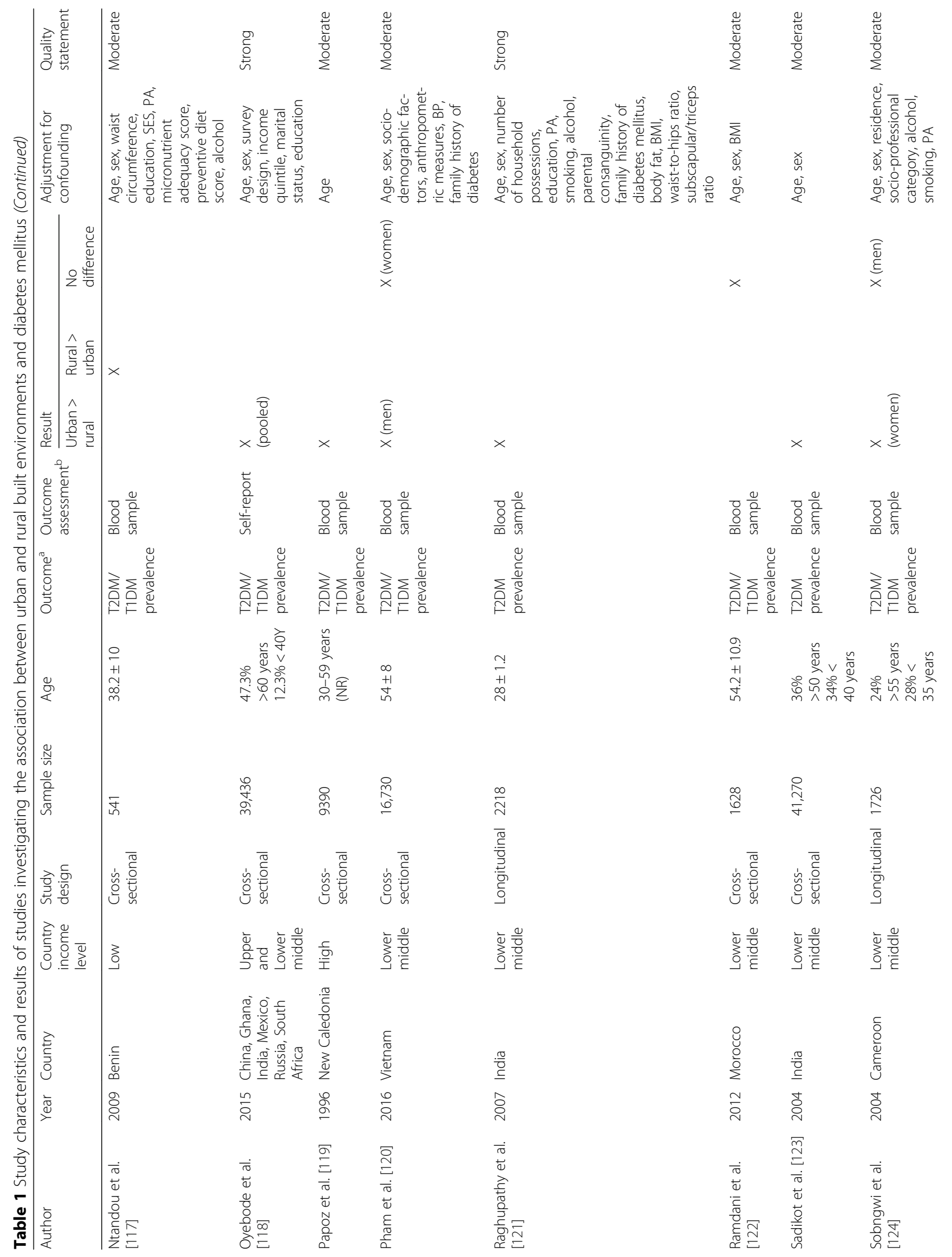




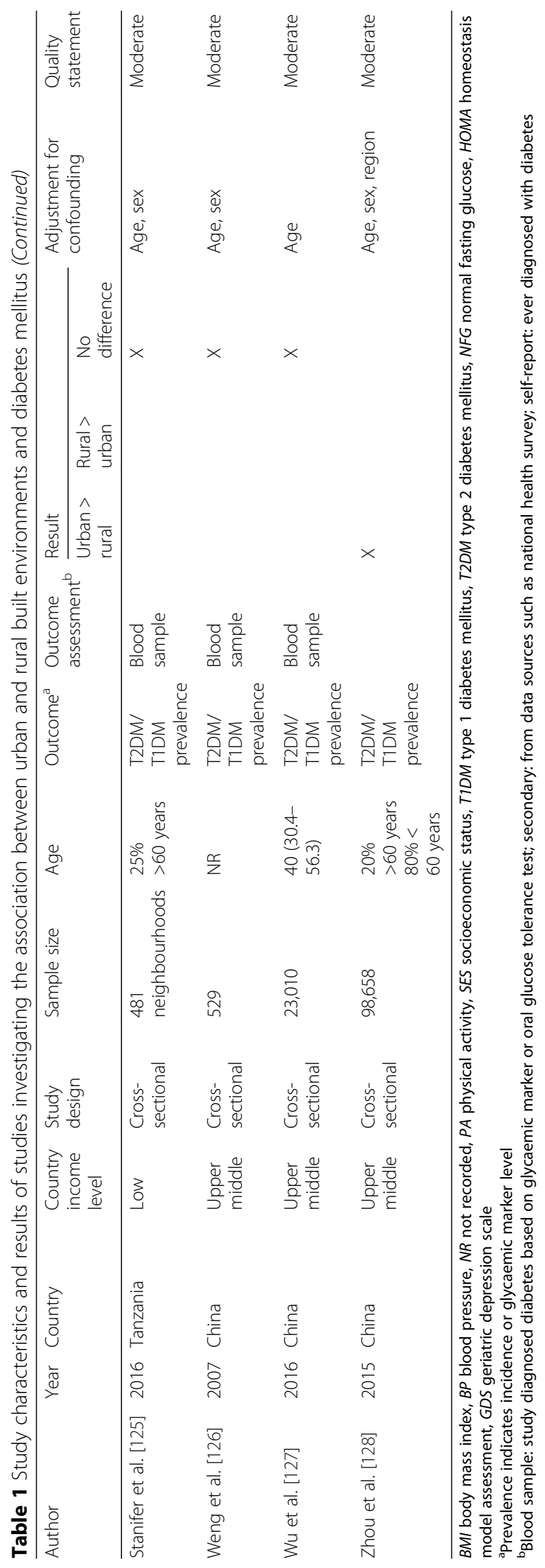




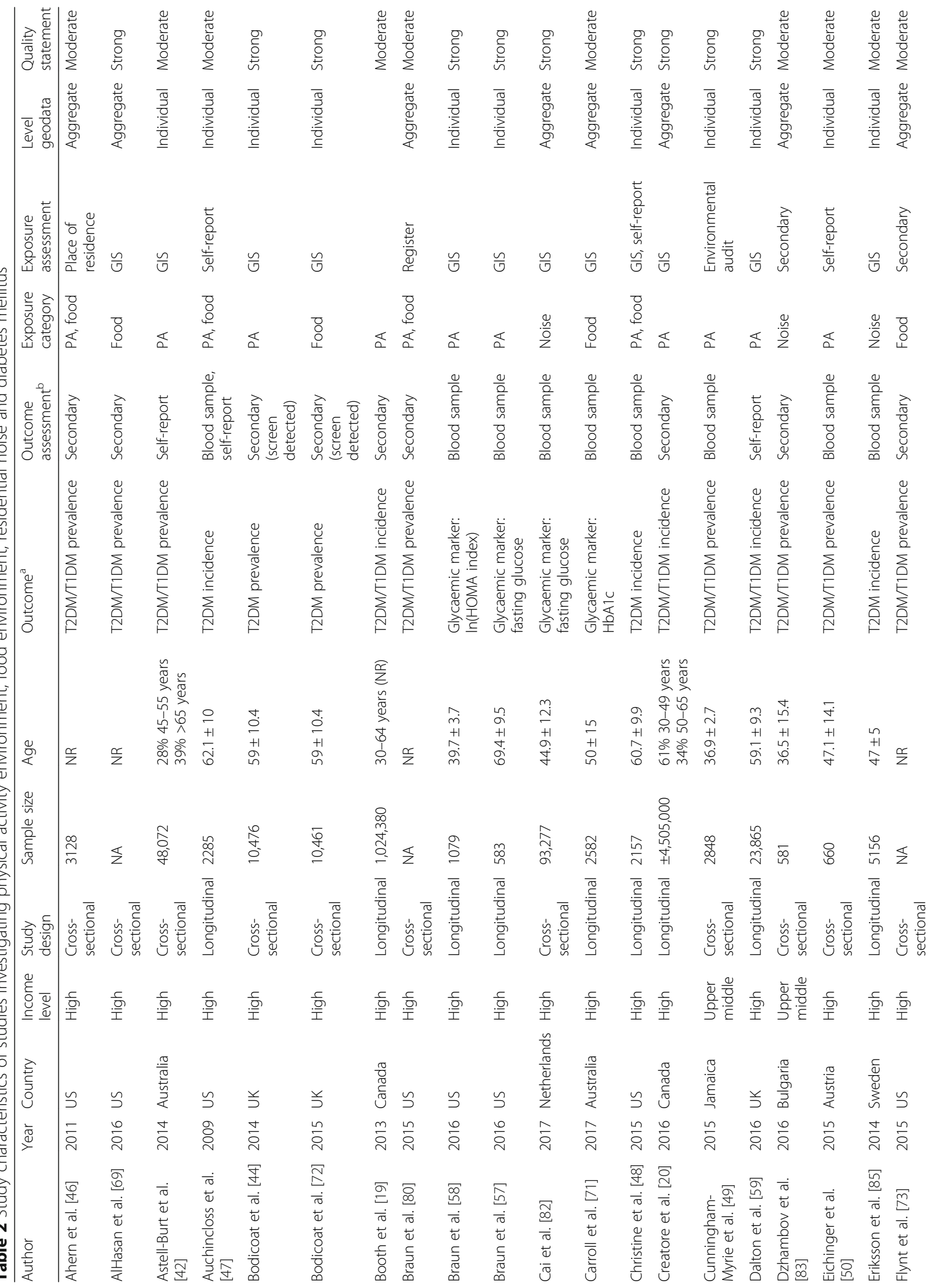




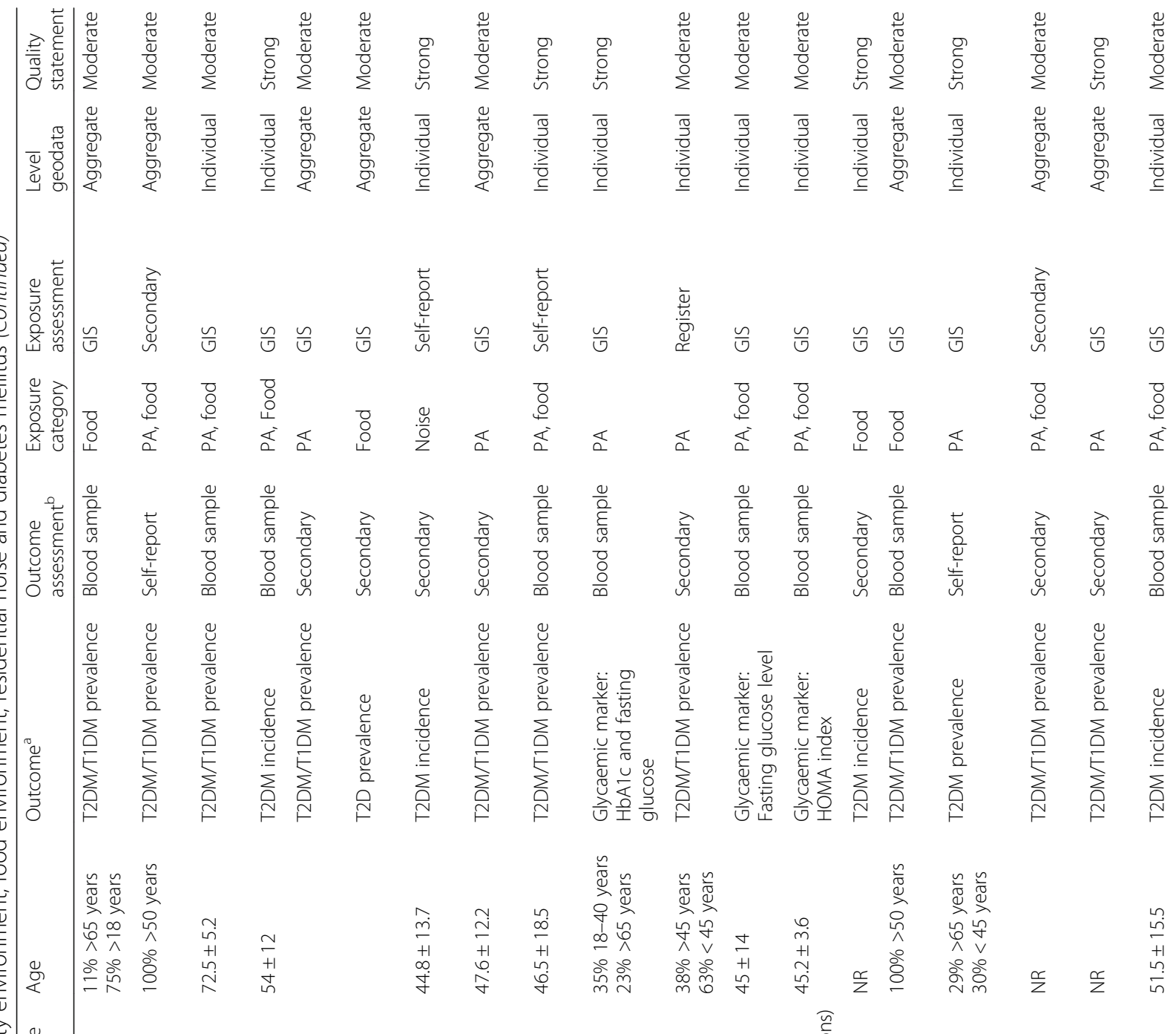

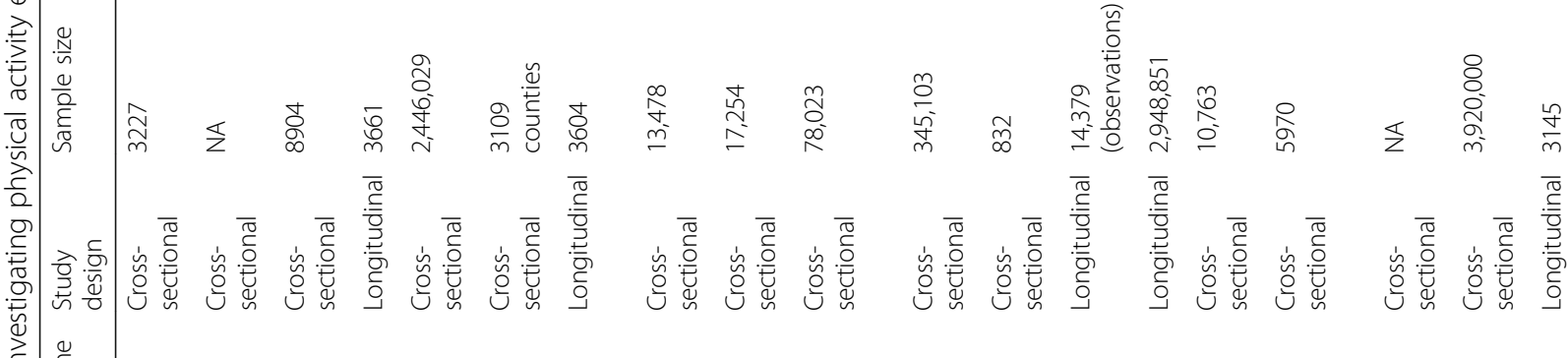
旁离总

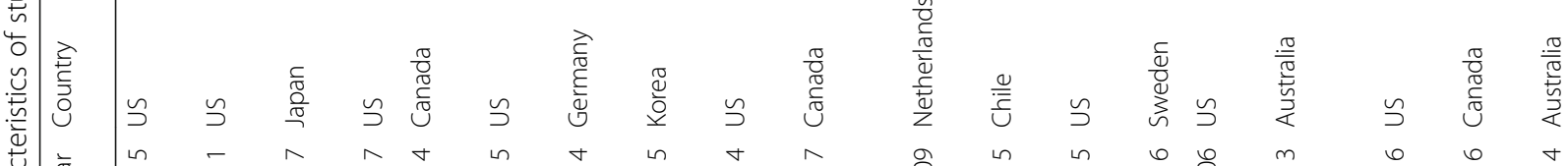
莡

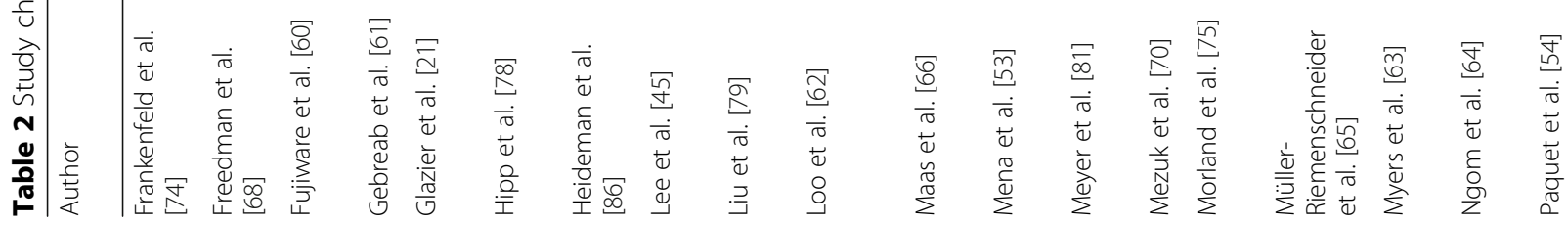




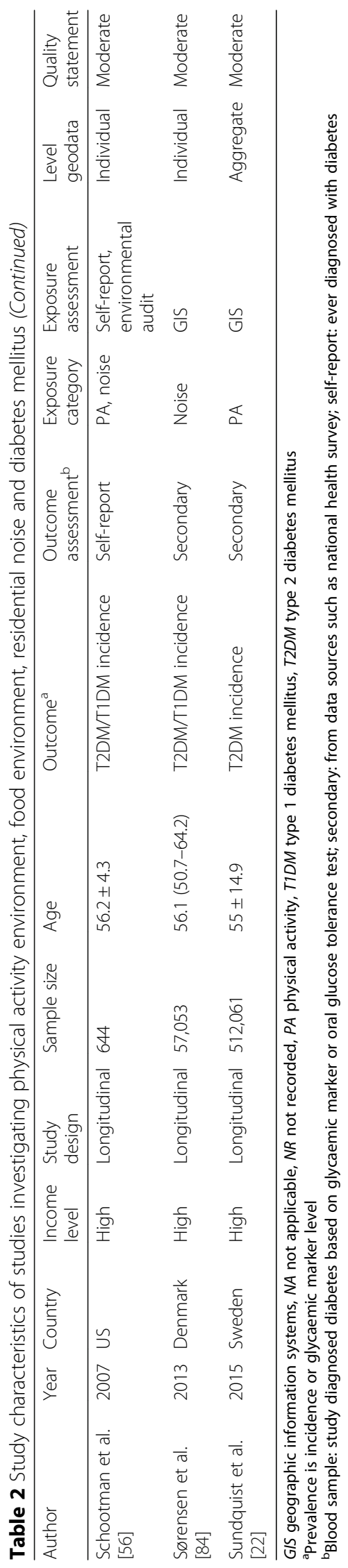


a

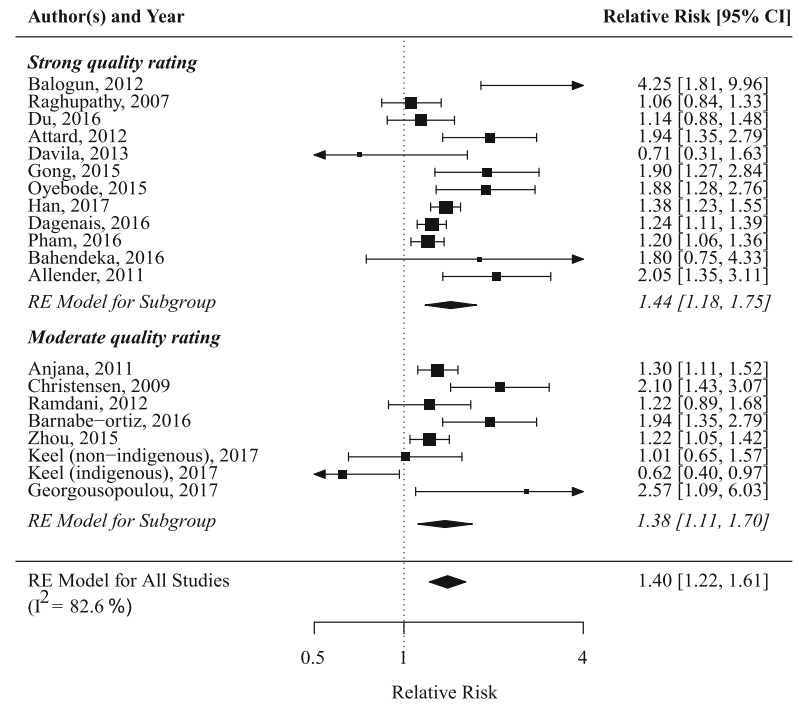

C

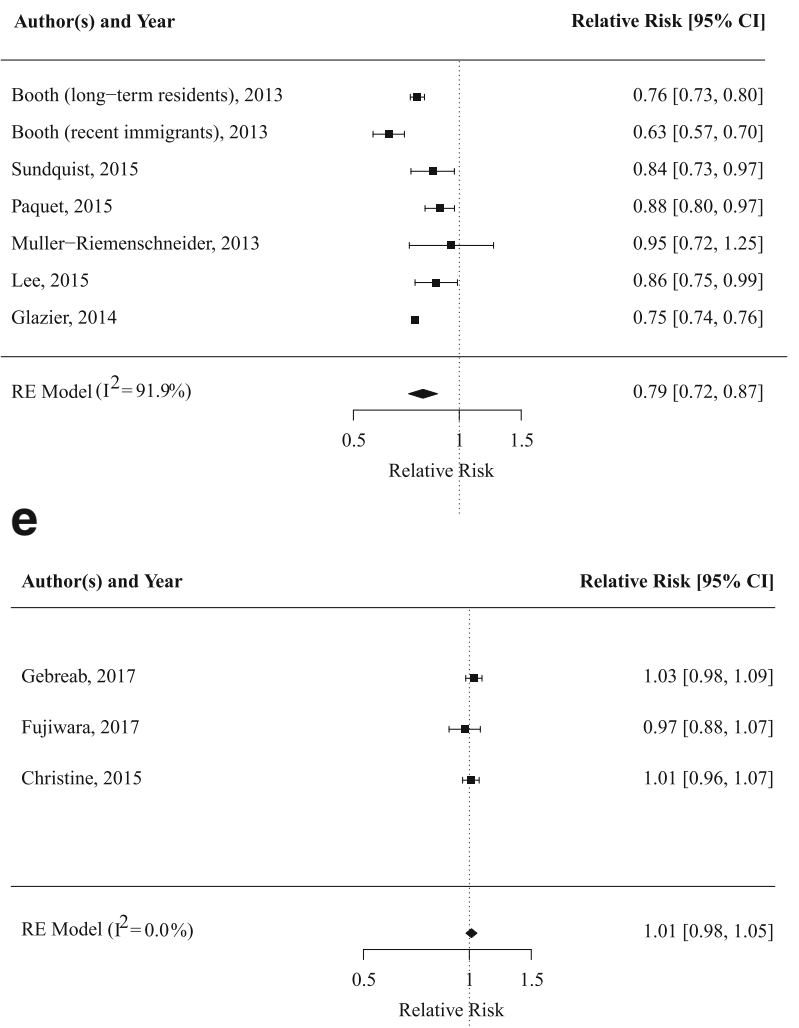

b

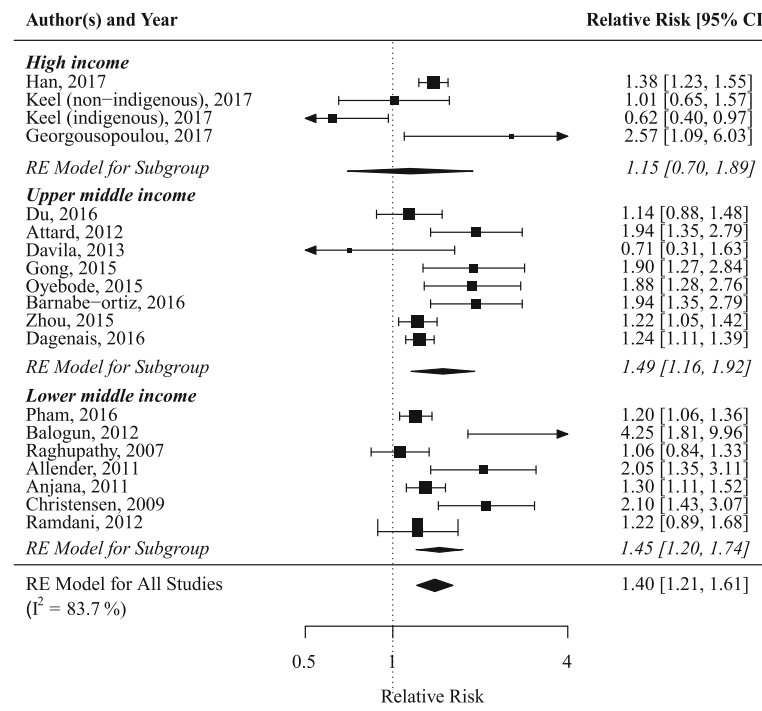

d

Author(s) and Year

Relative Risk [95\% CI]

\begin{tabular}{lll}
\hline Fujiware, 2017 & $1.00[0.86,1.15]$ \\
Ngom, 2016 & $1.09[1.04,1.14]$ \\
Dalton, 2016 & $0.81[0.66,1.00]$ \\
Maas, 2009 & $0.84[0.83,0.85]$ \\
Bodicoat, 2014 & $0.53[0.35,0.81]$ \\
Astell-Burt, 2014 & $0.94[0.85,1.03]$ \\
\hline RE Model $\left(1^{2}=95.1 \%\right)$ & $0.90[0.79,1.03]$
\end{tabular}

f

Author(s) and Yea Relative Risk $[95 \%$ CI]

Heideman, 2014

Sorensen, 2013

Dzhambov, 2016

Eriksson, 2014

$1.97[1.07,3.63]$

$0.93[0.88,0.98]$

$4.49[1.38,14.60]$

$0.94[0.33,2.69]$

RE Model $\left(\mathrm{l}^{2}=75.8 \%\right)$

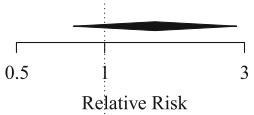

$1.49[0.78,2.82]$

Fig. 2 Forest plots of meta-analysis of the association between built environmental characteristics and T2DM risk/prevalence. a Urban vs. rural environments, stratified for study quality. b Urban vs. rural environments, stratified for country income level. c Walkability. d Green space. e Grocery stores. $\mathbf{f}$ Noise. T2DM type 2 diabetes mellitus. RE model random effects model

strong quality rating [48]. Two studies focused on the perceived availability of healthy foods, rather than objectively measured availability. One study observed greater self-reported availability of healthy food resources to be associated with lower T2DM risk [47]. The second study assessed perceived availability, objective availability and a combination of the two, of which only perceived availability was associated with a lower 
T2DM risk [48]. Another study found no association between the presence of food deserts and T2DM prevalence [78].

Three studies used a ratio of unhealthful food stores to more healthful food stores, such as the Relative Food Environment Index (RFEI), with a higher value indicating an unhealthier food environment. One study received a strong quality rating [70]. This study observed that a higher ratio, i.e. a relatively unhealthier food environment, was associated with a higher risk of T2DM. Two studies did not observe consistent associations between RFEI and T2DM risk [54, 74].

Six studies used composite measures of physical activity and food-related built environmental characteristics (Tables 2 and 3, and Additional file 4). One study received a strong quality rating [79]. A summary score indicating the presence of more healthy food resources and physical activity resources was associated with lower T2DM incidence [47]. Furthermore, residing in a neighbourhood with physical and social-environmental disadvantages was associated with higher T2DM prevalence [79]. Clusters of large metropolitan counties, characterised by low population density, median income, low socioeconomic status index and greater access to food observed less T2DM [73]. Finally, no association was observed between vibrancy index, density and obesogenicity clusters and T2DM risk/prevalence [68, 80, 81].

Four studies investigated the association between residential noise and T2DM risk/prevalence. One study received a strong quality rating [82]. All studies observed that higher exposure to residential noise was associated with increased T2DM risk/prevalence [82-85]. In metaanalyses of four studies [83-86], higher exposure to residential noise was not associated with T2DM risk/prevalence (1.49; 95\% CI, 0.78-2.82, $I^{2}=75.8 \%$ ).

\section{Discussion}

This systematic review investigated evidence for the association between built environmental characteristics, related to lifestyle behaviours, and T2DM risk/prevalence, worldwide. The association between built environmental characteristics and T2DM risk/prevalence has been investigated a fair amount, with 84 studies on the subject, although for our review, 23 of these studies were excluded due to their low quality ratings. Urbanisation was associated with a higher T2DM risk/prevalence. The evidence for an association between the physical activity environment and T2DM risk was more consistent than it was for the food environment. Higher neighbourhood walkability was associated with lower T2DM risk and more green space tended to be associated with lower T2DM risk.

First, we observed that residing in urban areas was associated with higher T2DM risk/prevalence, in line with the findings of the IDF diabetes atlas [8] and a recent meta-analysis for South East Asia. Urbanisation is a process in which inhabitants of a particular region increasingly move to more densely populated areas. Urbanisation is a broad operationalisation of the built environment and includes a range of characteristics, such as higher availability of food, facilities, and infrastructure. In general, previous reviews have observed conflicting results for urbanisation $[4,5,8]$. Urbanisation has consistently been associated with less physical activity and unhealthier dietary habits, but also with higher total walking and cycling for transportation $[4,5,8]$. The observed heterogeneity in terms of results might be due to the variety of definitions used to classify an urban area, which is distinct for different countries and studies. To account for this, we stratified our analyses by country income level [18], and the majority of studies (38 out of 60) were conducted in middle-income countries, which reduces the heterogeneity in the studies included. It must be recognised that considerable heterogeneity in definitions of urban vs. rural exists beyond stratification on country income level. Across countries with the same country income level, there is large variety of what urban or rural areas may look like and the populations that reside in these areas. At present, there is no homogeneous and generally accepted definition of urban or rural areas and the majority of studies did not include a definition that was used to make this classification.

Second, the present study provides consistent evidence for an association between the built physical activity environment and T2DM risk/prevalence. Higher walkability and availability of green space were most consistently associated with lower T2DM risk/prevalence. Our results for urbanisation seem contradictory to the lower T2DM risk/prevalence associated with greater neighbourhood walkability, since greater walkability is often observed in more urbanised environments [5]. These seemingly contradictory results could be explained by the underrepresentation of high-income countries in the urban to rural comparison studies, and the overrepresentation of these countries in walkability studies. The (perceived) walkability of urban areas also varies across different parts of the world. So, whereas walkability may be a feature of cities in high-income regions, this may not be the case in cities in lower-income regions. Furthermore, urbanisation is a much broader construct than walkability, and even within one urban area, walkability may differ between or even within neighbourhoods. In addition, other urbanisation-related issues, besides walkability, may be more important, such as other physical activity environment characteristics and the food environment, which counterbalance the effects of walkability in urban areas. These results would suggest that certain aspects of the built food environment were associated 
Table 3 Study results of studies investigating physical activity environment, food environment, residential noise and diabetes mellitus

\begin{tabular}{|c|c|c|c|c|}
\hline Author & Exposure & Study result & $\begin{array}{l}95 \% \text { confidence interval } \\
\text { or } p \text { value }\end{array}$ & Adjustment for confounding \\
\hline \multirow[t]{9}{*}{ Ahern et al., 2011 [46] } & Food environment: & Beta (SE) & & \multirow[t]{9}{*}{ Age, obesity rate } \\
\hline & $\begin{array}{l}\text { 1. Percentage of } \\
\text { households with } \\
\text { no car living more } \\
\text { than } 1 \text { mile from } \\
\text { a grocery store }\end{array}$ & 1. $0.07(0.01)$ & 1. $P<0.001$ & \\
\hline & $\begin{array}{l}\text { 2. Fast-food restaurants } \\
\text { per } 1000\end{array}$ & 2. $0.41(0.07)$ & 2. $P<0.001$ & \\
\hline & $\begin{array}{l}\text { 3. Full service restaurants } \\
\text { per } 1000\end{array}$ & 3. $-0.15(0.04)$ & 3. $P<0.01$ & \\
\hline & $\begin{array}{l}\text { 4. Grocery stores } \\
\text { per } 1000\end{array}$ & 4. $-0.37(0.09)$ & 4. $P<0.001$ & \\
\hline & $\begin{array}{l}\text { 5. Convenience stores } \\
\text { per } 1000\end{array}$ & 5. $0.30(0.06)$ & 5. $P<0.001$ & \\
\hline & $\begin{array}{l}\text { 6. Direct money made } \\
\text { from farm sales per capita }\end{array}$ & 6. $-0.01(0.02)$ & 6. $P<0.01$ & \\
\hline & PA environment: & & & \\
\hline & $\begin{array}{l}\text { 7. Recreational facilities } \\
\text { per } 1000\end{array}$ & 7. $-0.12(0.21)$ & 7. NS & \\
\hline \multirow[t]{5}{*}{ AlHasan et al., 2016 [69] } & Food outlet density: & Beta (SE) & & \multirow{5}{*}{$\begin{array}{l}\text { Age, obesity, PA, recreation } \\
\text { facility density, unemployed, } \\
\text { education, household with } \\
\text { no cars and limited } \\
\text { access to stores, race }\end{array}$} \\
\hline & $\begin{array}{l}\text { 1. Fast-food restaurant } \\
\text { density per } 1000 \text { residents }\end{array}$ & 1. $-0.55(0.90)$ & 1. NS & \\
\hline & $\begin{array}{l}\text { 2. Convenience } \\
\text { store density }\end{array}$ & 2. $0.89(0.86)$ & 2. NS & \\
\hline & 3. Super store density & 3. $-0.4(11.66)$ & 3. NS & \\
\hline & 4. Grocery store density & 4. $-3.7(2.13)$ & 4. NS & \\
\hline \multirow[t]{3}{*}{ Astell-Burt et al., 2014 [42] } & Green space (percent): & OR: & & \multirow{3}{*}{$\begin{array}{l}\text { Age, sex, couple status, } \\
\text { family history, country } \\
\text { of birth, language spoken } \\
\text { at home, weight, psychological } \\
\text { distress, smoking status, } \\
\text { hypertension, diet, walking, } \\
\text { MVPA, sitting, economic } \\
\text { status, annual income, } \\
\text { qualifications, neighbourhood } \\
\text { affluence, geographic remoteness }\end{array}$} \\
\hline & 1. $>81$ & 1. 0.94 & 1. $0.85-1.03$ & \\
\hline & 2. $0-20$ & 2. 1 & 2. NA & \\
\hline \multirow[t]{4}{*}{ Auchincloss et al., 2009 [47] } & $\begin{array}{l}\text { Neighbourhood } \\
\text { resources: }\end{array}$ & HR: & & \multirow{4}{*}{$\begin{array}{l}\text { Age, sex, family history, } \\
\text { income, assets, education, } \\
\text { ethnicity, alcohol, smoking, } \\
\text { PA, diet, BMI }\end{array}$} \\
\hline & $\begin{array}{l}\text { 1. Healthy food } \\
\text { resources }\end{array}$ & 1. 0.63 & 1. $0.42-0.93$ & \\
\hline & 2. PA resources & 2. 0.71 & 2. $0.48-1.05$ & \\
\hline & 3. Summary score & 3. 0.64 & 3. $0.44-0.95$ & \\
\hline \multirow[t]{3}{*}{ Bodicoat et al., 2014 [44] } & Green space (percent) & OR: & & \multirow{3}{*}{$\begin{array}{l}\text { Age, sex, area social deprivation } \\
\text { score, urban/rural status, BMI, PA, } \\
\text { fasting glucose, } 2 \text { h glucose, } \\
\text { total cholesterol }\end{array}$} \\
\hline & 1. Least green space (Q1) & 1.1 & 1. NA & \\
\hline & 2. Most green space (Q4) & 2. 0.53 & 2. $0.35-0.82$ & \\
\hline \multirow[t]{3}{*}{ Bodicoat et al., 2015 [72] } & & OR: & & \multirow{3}{*}{$\begin{array}{l}\text { Age, sex, area social } \\
\text { deprivation score, urban/rural } \\
\text { status, ethnicity, PA }\end{array}$} \\
\hline & $\begin{array}{l}\text { 1. Number of fast-food } \\
\text { outlets (per } 2 \text { ) }\end{array}$ & 1. 1.02 & 1. $1.00-1.04$ & \\
\hline & $\begin{array}{l}\text { 2. Density of fast-food } \\
\text { outlet (per } 200 \text { residents) }\end{array}$ & 2. 13.84 & 2. $1.60-119.6$ & \\
\hline \multirow[t]{5}{*}{ Booth et al., 2013 [19] } & Walkability: & HR: & & \multirow[t]{5}{*}{ Age, sex, income } \\
\hline & Men & & & \\
\hline & Recent immigrants & & & \\
\hline & 1. Least walkable quintile & 1. 1.58 & 1. $1.42-1.75$ & \\
\hline & 2. Most walkable quintile & 2. 1 & 2. NA & \\
\hline
\end{tabular}


Table 3 Study results of studies investigating physical activity environment, food environment, residential noise and diabetes mellitus (Continued)

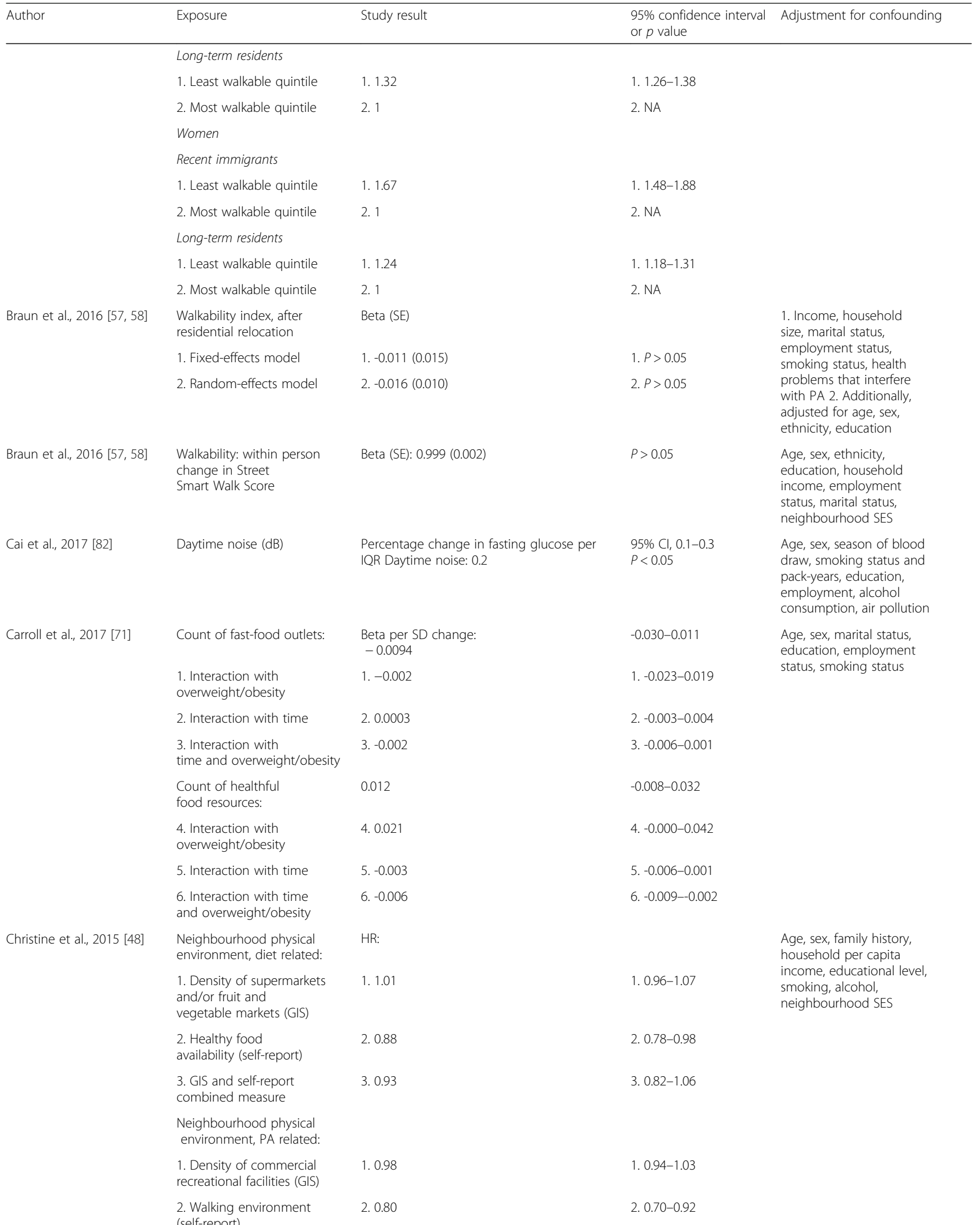


Table 3 Study results of studies investigating physical activity environment, food environment, residential noise and diabetes mellitus (Continued)

\begin{tabular}{|c|c|c|c|c|}
\hline Author & Exposure & Study result & $\begin{array}{l}95 \% \text { confidence interval } \\
\text { or } p \text { value }\end{array}$ & Adjustment for confounding \\
\hline & $\begin{array}{l}\text { 3. GIS and self-report } \\
\text { combined measure }\end{array}$ & 3. 0.81 & 3. $0.68-0.96$ & \\
\hline \multirow[t]{3}{*}{ Creatore et al., 2016 [20] } & Walkability: & $\begin{array}{l}\text { Absolute incidence } \\
\text { rate difference } \\
\text { over } 12 \text { years } \mathrm{FU} \text { : }\end{array}$ & & \multirow[t]{3}{*}{$\begin{array}{l}\text { Age, sex, area } \\
\text { income, ethnicity }\end{array}$} \\
\hline & $\begin{array}{l}\text { 1. Low walkable } \\
\text { neighbourhoods (Q1) }\end{array}$ & 1. -0.65 & 1. $-1.65-0.39$ & \\
\hline & $\begin{array}{l}\text { 2. High walkable } \\
\text { neighbourhoods over (Q5) }\end{array}$ & 2. -1.5 & 2. $-2.6--0.4$ & \\
\hline \multirow[t]{7}{*}{$\begin{array}{l}\text { Cunningham-Myrie et al., } \\
2015 \text { [49] }\end{array}$} & $\begin{array}{l}\text { Neighbourhood } \\
\text { characteristics: }\end{array}$ & OR: & & \multirow[t]{7}{*}{$\begin{array}{l}\text { Age, sex, district, fruit } \\
\text { and vegetable intake }\end{array}$} \\
\hline & $\begin{array}{l}\text { 1. Neighbourhood } \\
\text { infrastructure }\end{array}$ & 1. 1.02 & 1. $0.95-1.1$ & \\
\hline & $\begin{array}{l}\text { 2. Neighbourhood disorder } \\
\text { score }\end{array}$ & 2. 0.99 & 2. $0.95-1.03$ & \\
\hline & 3. Home disorder score & 3. 1 & 3. $0.96-1.03$ & \\
\hline & $\begin{array}{l}\text { 4. Recreational space } \\
\text { in walking distance }\end{array}$ & 4. 1.12 & 4. $0.86-1.45$ & \\
\hline & $\begin{array}{l}\text { 5. Recreational } \\
\text { space availability }\end{array}$ & 5. 1.01 & 5. $0.77-1.32$ & \\
\hline & 6. Perception of safety & 6. 0.99 & 6. $0.88-1.11$ & \\
\hline \multirow[t]{4}{*}{ Dalton et al., 2016 [59] } & Green space: & HR: & & \multirow{4}{*}{$\begin{array}{l}\text { Age, sex, BMI, parental } \\
\text { diabetes, SES Effect } \\
\text { modification by urban-rural } \\
\text { status and SES was } \\
\text { investigated, but } \\
\text { association was } \\
\text { not moderated by either }\end{array}$} \\
\hline & 1. Least green space (Q1) & 1. 1 & 1. NA & \\
\hline & 2. Most green space (Q4) & 2. 0.81 & 2. $0.65-0.99$ & \\
\hline & 3. Mediation by PA & 3. 0.96 & 3. $0.88-1.06$ & \\
\hline \multirow[t]{3}{*}{ Dzhambov et al., 2016 [83] } & $\begin{array}{l}\text { Day-evening-night } \\
\text { equivalent sound level: }\end{array}$ & OR: & & \multirow{3}{*}{$\begin{array}{l}\text { Age, sex, fine particulate } \\
\text { matter, benzo alpha pyrene, } \\
\text { BMI, family history of } \\
\text { T2DM, subjective sleep } \\
\text { disturbance, bedroom location }\end{array}$} \\
\hline & 1. 51-70 decibels & 1. 1 & 1. NA & \\
\hline & 2. 71-80 decibels & 2. 4.49 & 2. $1.39-14.7$ & \\
\hline \multirow[t]{8}{*}{ Eichinger et al., 2015 [50] } & $\begin{array}{l}\text { Characteristics of built } \\
\text { residential environment: }\end{array}$ & Beta: & & \multirow[t]{8}{*}{ Age, sex, individual-level SES } \\
\hline & $\begin{array}{l}\text { 1. Perceived distance } \\
\text { to local facilities }\end{array}$ & 1. 0.006 & 1. $P<0.01$ & \\
\hline & $\begin{array}{l}\text { 2. Perceived availability } \\
\text { /maintenance of } \\
\text { cycling/walking infrastructure }\end{array}$ & 2. NS & & \\
\hline & 3. Perceived connectivity & 3. NS & & \\
\hline & $\begin{array}{l}\text { 4. Perceived safety with } \\
\text { regards to traffic }\end{array}$ & 4. NS & & \\
\hline & 5. perceived safety from crime & 5. NS & & \\
\hline & $\begin{array}{l}\text { 6. Neighbourhood as } \\
\text { pleasant environment } \\
\text { for walking/cycling }\end{array}$ & 6. NS & & \\
\hline & $\begin{array}{l}\text { 7. Presence of trees } \\
\text { along the streets }\end{array}$ & 7. NS & & \\
\hline \multirow[t]{3}{*}{ Eriksson et al., 2014 [85] } & Aircraft noise level: & OR: & & \multirow{3}{*}{$\begin{array}{l}\text { Age, sex, family history, } \\
\text { SES based on education, } \\
\text { PA, smoking, alcohol, } \\
\text { annoyance due to noise }\end{array}$} \\
\hline & 1. $<50 \mathrm{~dB}$ & 1. 1 & 1. NA & \\
\hline & 2. $\geq 55 \mathrm{~dB}$ & 2. 0.94 & 2. $0.33-2.70$ & \\
\hline Flynt et al., 2015 [73] & $\begin{array}{l}\text { Clusters (combination } \\
\text { of number of counties, } \\
\text { urban-rural classification, } \\
\text { population density, } \\
\text { income, SES, access } \\
\text { to food stores, obesity } \\
\text { rate, diabetes rate): }\end{array}$ & $\begin{array}{l}\text { Median standardised } \\
\text { diabetes mellitues rate: }\end{array}$ & IQR: & - \\
\hline
\end{tabular}


Table 3 Study results of studies investigating physical activity environment, food environment, residential noise and diabetes mellitus (Continued)

\begin{tabular}{|c|c|c|c|c|}
\hline Author & Exposure & Study result & $\begin{array}{l}95 \% \text { confidence interval } \\
\text { or } p \text { value }\end{array}$ & Adjustment for confounding \\
\hline & 1 & 1. 0 & 1. $-0.05-0.7$ & \\
\hline & 2 & 2. 0 & 2. $-0.04-0.7$ & \\
\hline & 3 & 3.0 & 3. $-0.08-0.01$ & \\
\hline & 4 & 4. -0.04 & 4. $-1.01-0.6$ & \\
\hline & 5 & 5. -0.08 & $\begin{array}{l}\text { 5. }-1.5--0.04 \\
\text { ANOVA: } p<0.001\end{array}$ & \\
\hline \multirow[t]{7}{*}{ Frankenfeld et al., 2015 [74] } & RFEl $\leq 1$ clusters: & Predicted prevalence: & & \multirow[t]{7}{*}{ Demographic and SES variables } \\
\hline & 1. Grocery stores & 1. 7.1 & 1. $6.3-7.9$ & \\
\hline & 2. Restaurants & 2. 5.9 & 2. $5.0-6.8, p<0.01$ & \\
\hline & 3. Specialty foods & 3. 6.1 & 3. $5.0-7.2, p<0.01$ & \\
\hline & RFEI >1: & & & \\
\hline & 4. Restaurants and fast-food & 4. 6.0 & 4. $4.9-7.1, p<0.01$ & \\
\hline & 5. Convenience stores & 5. 6.1 & 5. 4.9-7.3, $p<0.01$ & \\
\hline \multirow[t]{8}{*}{ Freedman et al., 2011 [68] } & Built environment: & OR: & & \multirow{8}{*}{$\begin{array}{l}\text { Age, ethnicity, marital status, } \\
\text { region of residence, smoking, } \\
\text { education, income, childhood } \\
\text { health, childhood SES, region } \\
\text { of birth, neighbourhood scales }\end{array}$} \\
\hline & Men: & & & \\
\hline & $\begin{array}{l}\text { 1. Connectivity ( } 2000 \\
\text { Topologically Integrated }\end{array}$ & 1. 1.06 & 1. $0.86-1.29$ & \\
\hline & $\begin{array}{l}\text { Geographic Encoding } \\
\text { and Referencing system) }\end{array}$ & 2. 1.05 & 2. $0.89-1.24$ & \\
\hline & $\begin{array}{l}\text { 2. Density (number of } \\
\text { food stores, restaurants, } \\
\text { housing units per square mile) }\end{array}$ & & & \\
\hline & Women: & & & \\
\hline & 3. Connectivity & 3. 1.01 & 3. $0.84-1.20$ & \\
\hline & 4. Density & 4. 0.99 & 4. $0.99-1.17$ & \\
\hline \multirow[t]{3}{*}{ Fujiware et al., 2017 [60] } & $\begin{array}{l}\text { Count within } \\
\text { neighbourhood unit } \\
\left(\text { mean } 6.31 \pm 3.9 \mathrm{~km}^{2}\right)\end{array}$ & OR per IQR increase: & & \multirow{3}{*}{$\begin{array}{l}\text { Age, sex, marital status, } \\
\text { household number, } \\
\text { income, working status, } \\
\text { drinking, smoking, } \\
\text { vegetable consumption, } \\
\text { walking, going-out behaviour, } \\
\text { frequency of meeting, } \\
\text { BMl, depression }\end{array}$} \\
\hline & 1. Grocery stores & 1. 0.97 & 1. $0.88-1.08$ & \\
\hline & 2. Parks & 2. 1.16 & 2. $1-1.34$ & \\
\hline \multirow[t]{4}{*}{ Gebreab et al., 2017 [61] } & Density within 1-mile buffer: & HR: & & \multirow{4}{*}{$\begin{array}{l}\text { Age, sex, family history } \\
\text { of diabetes, SES, smoking, } \\
\text { alcohol consumption, } \\
\text { physical activity, diet }\end{array}$} \\
\hline & 1. Favourable food stores & 1. 1.03 & 1. $0.98-1.09$ & \\
\hline & 2. Unfavourable food stores & 2. 1.07 & 2. $0.99-1.16$ & \\
\hline & 3. PA resources & 3. 1.03 & 3. $0.98-1.09$ & \\
\hline \multirow[t]{8}{*}{ Glazier et al., 2014 [21] } & Walkability index: & Rate ratio: & & \multirow[t]{8}{*}{ Age, sex } \\
\hline & 1. Q1 & 1. 1 & 1. NA & \\
\hline & 2. Q5 & 2. 1.33 & 2. $1.33-1.33$ & \\
\hline & Index components: & & & \\
\hline & $\begin{array}{l}\text { 1. Population } \\
\text { density (Q1: Q5) }\end{array}$ & 1. 1.16 & 1. $1.16-1.16$ & \\
\hline & 2. Residential density (Q1: Q5) & 2. 1.33 & 2. $1.33-1.33$ & \\
\hline & 3. Street connectivity (Q1: Q5) & 3. 1.38 & 3. $1.38-1.38$ & \\
\hline & $\begin{array}{l}\text { 4. Availability of } \\
\text { walkable destinations (Q1: Q5) }\end{array}$ & 4. 1.26 & 4. $1.26-1.26$ & \\
\hline \multirow[t]{3}{*}{ Heidemann et al., 2014 [86] } & $\begin{array}{l}\text { Residential } \\
\text { traffic intensity: }\end{array}$ & OR: & & \multirow{3}{*}{$\begin{array}{l}\text { Age, sex, smoking, passive } \\
\text { smoking, heating of house, } \\
\text { education, BMI, waist } \\
\text { circumference, PA, family history }\end{array}$} \\
\hline & 1. No traffic & 1. 1 & 1. NA & \\
\hline & 2. Extreme traffic & 2. 1.97 & 2. $1.07-3.64$ & \\
\hline Hipp et al., 2015 [78] & Food deserts & Correlation: NR & NS & - \\
\hline
\end{tabular}


Table 3 Study results of studies investigating physical activity environment, food environment, residential noise and diabetes mellitus (Continued)

\begin{tabular}{|c|c|c|c|c|}
\hline Author & Exposure & Study result & $\begin{array}{l}95 \% \text { confidence interval } \\
\text { or } p \text { value }\end{array}$ & Adjustment for confounding \\
\hline \multirow[t]{3}{*}{ Lee et al., 2015 [45] } & Walkability: & OR: & & \multirow{3}{*}{$\begin{array}{l}\text { Age, sex, smoking, } \\
\text { alcohol, income level }\end{array}$} \\
\hline & 1. Community 1 & 1. 1 & 1. NA & \\
\hline & 2. Community 2 & 2. 0.86 & 2. $0.75-0.99$ & \\
\hline \multirow[t]{4}{*}{ Loo et al., 2017 [62] } & \multirow{4}{*}{$\begin{array}{l}\text { Walkability (walk score) } \\
\text { Difference between } \\
\text { Q1 and Q4 }\end{array}$} & Beta for $\mathrm{HbA1C}$ : & & \multirow{4}{*}{$\begin{array}{l}\text { Age, sex, current smoking } \\
\text { status, BMI, relevant } \\
\text { medications and medical } \\
\text { diagnoses, neighbourhood } \\
\text { violent crime rates and } \\
\text { neighbourhood indices } \\
\text { of material deprivation, } \\
\text { ethnic concentration, } \\
\text { dependency, } \\
\text { residential instability }\end{array}$} \\
\hline & & 1. -0.06 & 1. $-0.11-0.02$ & \\
\hline & & Beta for fasting glucose: & & \\
\hline & & 2. 0.03 & 2. $-0.04-0.1$ & \\
\hline \multirow[t]{3}{*}{ Maas et al., 2009 [66] } & Green space: & OR: & & \multirow{3}{*}{$\begin{array}{l}\text { Demographic and } \\
\text { socioeconomic } \\
\text { characteristics, urbanicity }\end{array}$} \\
\hline & 1. Q1 & 1. 1 & 1. NA & \\
\hline & 2. Q4 & 2. 0.84 & 2. $0.83-0.85$ & \\
\hline \multirow[t]{3}{*}{ Mena et al., 2015 [53] } & & Correlation: & & \multirow[t]{3}{*}{-} \\
\hline & 1. Distance to parks & 1. NR & 1. NA & \\
\hline & 2. Distance to markets & 2. -0.094 & 2. $P<0.05$ & \\
\hline Mezuk et al., 2016 [70] & $\begin{array}{l}\text { Ratio of the number } \\
\text { of health-harming food } \\
\text { outlets to the total } \\
\text { number of food outlets } \\
\text { within a } 1000-m \\
\text { buffer of each person }\end{array}$ & OR per km²: 2.11 & $1.57-2.82$ & $\begin{array}{l}\text { Age, sex, education, } \\
\text { household income }\end{array}$ \\
\hline \multirow[t]{4}{*}{ Morland et al., 2006 [75] } & Presence of: & Prevalence ratio: & & \multirow{4}{*}{$\begin{array}{l}\text { Age, sex, income, education, } \\
\text { ethnicity, food stores } \\
\text { and service places, PA }\end{array}$} \\
\hline & 1. Supermarkets & 1. 0.96 & 1. $0.84-1.1$ & \\
\hline & 2. Grocery stores & 2. 1.11 & 2. $0.99-1.24$ & \\
\hline & 3. Convenience stores & 3. 0.98 & 3. $0.86-1.12$ & \\
\hline \multirow{6}{*}{$\begin{array}{l}\text { Müller-Riemenschneider et } \\
\text { al., } 2013 \text { [65] }\end{array}$} & Walkability (1600 m buffer): & OR: & & \multirow{6}{*}{$\begin{array}{l}\text { Age, sex, education, household } \\
\text { income, marital status }\end{array}$} \\
\hline & 1. High walkability & 1. 0.95 & 1. $0.72-1.25$ & \\
\hline & 2. Low walkability & 2. 1 & 2. NA & \\
\hline & Walkability (800 m buffer): & & & \\
\hline & 3. High walkability & 3. 0.69 & 3. $0.62-0.90$ & \\
\hline & 4. Low walkability & 4. 1 & 4. NA & \\
\hline \multirow[t]{6}{*}{ Myers et al., 2017 [63] } & Physical activity: & Beta: & & \multirow[t]{6}{*}{ Age } \\
\hline & $\begin{array}{l}\text { 1. Recreation facilities } \\
\text { per } 1000\end{array}$ & 1. -0.457 & 1. $-0.809--0.104$ & \\
\hline & 2. Natural amenities (1-7) & 2. 0.084 & 2. $0.042-0.127$ & \\
\hline & Food: & & & \\
\hline & $\begin{array}{l}\text { 3. Grocery stores and } \\
\text { supercentres per } 1000\end{array}$ & 3. 0.059 & 3. $-0.09-0.208$ & \\
\hline & $\begin{array}{l}\text { 4. Fast-food restaurants } \\
\text { per } 1000\end{array}$ & 4. -0.032 & 4. $-0.125-0.062$ & \\
\hline \multirow[t]{3}{*}{ Ngom et al., 2016 [64] } & $\begin{array}{l}\text { Distance to } \\
\text { green space: }\end{array}$ & Prevalence ratio: & & \multirow[t]{3}{*}{$\begin{array}{l}\text { Age, sex, social and } \\
\text { environmental predictors }\end{array}$} \\
\hline & 1. Q1 (0-264 m) & 1. 1 & 1. NA & \\
\hline & 2. Q4 (774-27781 m) & 2. 1.09 & 2. $1.03-1.13$ & \\
\hline \multirow[t]{3}{*}{ Paquet et al., 2014 [54] } & $\begin{array}{l}\text { Built environment } \\
\text { attributes: }\end{array}$ & RR: & & \multirow{3}{*}{$\begin{array}{l}\text { Age, sex household } \\
\text { income, education, } \\
\text { duration of FU, area-level SES }\end{array}$} \\
\hline & 1. RFEI & 1. 0.99 & 1. $0.9-1.09$ & \\
\hline & 2. Walkability & 2. 0.88 & 2. $0.8-0.97$ & \\
\hline
\end{tabular}


Table 3 Study results of studies investigating physical activity environment, food environment, residential noise and diabetes mellitus (Continued)

\begin{tabular}{|c|c|c|c|c|}
\hline Author & Exposure & Study result & $\begin{array}{l}95 \% \text { confidence interval } \\
\text { or } p \text { value }\end{array}$ & Adjustment for confounding \\
\hline & 3. POS & & & \\
\hline & a. POS count & a. 1 & a. $0.92-1.08$ & \\
\hline & b. POS size & b. 0.75 & b. $0.69-0.83$ & \\
\hline & c. POS greenness & c. 1.01 & c. $0.9-1.13$ & \\
\hline & d. POS type & d. 1.09 & d. $0.97-1.22$ & \\
\hline \multirow[t]{11}{*}{ Schootman et al., 2007 [56] } & $\begin{array}{l}\text { Neighbourhood } \\
\text { conditions (objective): }\end{array}$ & OR: & & \multirow{11}{*}{$\begin{array}{l}\text { Age, sex, income, } \\
\text { perceived income } \\
\text { adequacy, education, } \\
\text { marital status, } \\
\text { employment, length } \\
\text { of time at present } \\
\text { address, own the home, area }\end{array}$} \\
\hline & 1. Housing conditions & 1. 1.11 & 1. $0.63-1.95$ & \\
\hline & $\begin{array}{l}\text { 2. Noise level from } \\
\text { traffic, industry, etc. }\end{array}$ & 2. 0.9 & 2. $0.48-1.67$ & \\
\hline & 3. Air quality & 3. 1.2 & 3. $0.66-2.18$ & \\
\hline & 4. Street and road quality & 4. 1.03 & 4. $0.56-1.91$ & \\
\hline & 5. Yard and sidewalk quality & 5. 1.05 & 5. $0.59-1.88$ & \\
\hline & $\begin{array}{l}\text { Neighbourhood } \\
\text { conditions (subjective): }\end{array}$ & & & \\
\hline & $\begin{array}{l}\text { 6. Fair-poor rating } \\
\text { of the neighbourhood }\end{array}$ & 6. 1.04 & 6. $0.58-1.84$ & \\
\hline & $\begin{array}{l}\text { 7. Mixed or terrible } \\
\text { feeling about the } \\
\text { neighbourhood }\end{array}$ & 7. 1.1 & 7. $0.6-2.02$ & \\
\hline & $\begin{array}{l}\text { 8. Undecided or not } \\
\text { at all attached to } \\
\text { the neighbourhood }\end{array}$ & 8. 0.68 & 8. $0.4-1.18$ & \\
\hline & $\begin{array}{l}\text { 9. Slightly unsafe-not } \\
\text { at all safe in } \\
\text { the neighbourhood }\end{array}$ & 9. 0.61 & 9. $0.35-1.06$ & \\
\hline \multirow[t]{3}{*}{ Sørensen et al., 2013 [84] } & $\begin{array}{l}\text { Exposure to road } \\
\text { traffic noise per } 10 \mathrm{~dB} \text { : }\end{array}$ & Incidence rate ratio: & & \multirow{3}{*}{$\begin{array}{l}\text { Age, sex, education, } \\
\text { municipality SES, } \\
\text { smoking status, } \\
\text { smoking intensity, } \\
\text { smoking duration, } \\
\text { environmental } \\
\text { tobacco smoke, } \\
\text { fruit intake, vegetable } \\
\text { intake, saturated fat } \\
\text { intake, alcohol, BMl, } \\
\text { waist circumference, } \\
\text { sports, walking, pollution }\end{array}$} \\
\hline & 1. At diagnosis & 1. 1.08 & 1. $1.02-1.14$ & \\
\hline & $\begin{array}{l}\text { 2. } 5 \text { years preceding } \\
\text { diagnosis }\end{array}$ & 2. 1.11 & 2. $1.05-1.18$ & \\
\hline \multirow[t]{3}{*}{ Sundquist et al., 2015 [22] } & Walkability: & OR: & & \multirow{3}{*}{$\begin{array}{l}\text { Age, sex, income, } \\
\text { education, } \\
\text { neighbourhood } \\
\text { deprivation }\end{array}$} \\
\hline & 1. D1 (low) & 1. 1.16 & 1. $1.00-1.34$ & \\
\hline & 2. D10 (high) & 2. 1 & 2. NA & \\
\hline
\end{tabular}

$B M I$ body mass index, CI Confidence interval, GIS graphical information system, HR hazard ratio, IQR interquartile range, NA not applicable, NR not reported, NS not significant, OR odds ratio, PA physical activity, MVPA moderate to vigorous physical activity, POS Public open space, RFEI Retail Food Environment Index, RR relative risk, $S D$ standard deviation, $S E$ standard error, SES socioeconomic status, FU follow-up

with a higher T2DM risk, but we could not find consistent evidence of this in our review.

An association between the built food environment and T2DM risk/prevalence was not consistently observed. The availability of fast-food and convenience stores and the perceived healthiness of the food environment tended to be associated with higher T2DM risk/ prevalence and lower T2DM risk/prevalence, respectively. However, due to heterogeneity in the studies, insufficient studies were available for meta-analysis, thus preventing us from drawing solid conclusions. The only possible meta-analyses were three studies including the density of grocery stores, but this confirmed that no significant associations could be observed. Also by reviewing the evidence, supermarkets and grocery stores and the RFEI were not associated with T2DM risk/prevalence. These findings are consistent with an earlier systematic review that reported that perceived availability was associated with healthy dietary behaviours [9], whereas objective measures of accessibility and availability of food environment yielded mixed results [9]. The association between the perceived environment and a 
healthier diet can be explained by not limiting the concept of environment to specific shops or locations, but rather to the participant's resources for healthy food, e.g. gardens and markets. On the other hand, perceptions may also reflect an individual's intentions and motivations rather than location alone. A difficulty with regard to establishing useful diet measures is that they are very heterogeneous and difficult to define. For instance, access to a supermarket is often seen as contributing to a healthy food environment, even though they are also sources of unhealthy products [9]. Establishing a comprehensive definition is further complicated because food can be bought in a variety of shops and locations that are not directly associated with food, e.g. at the counter of a pharmacy. The same heterogeneity was observed to a lesser extent in the built physical activity environment. For instance, infrastructure includes drivers for active transportation (sidewalks and cycling lanes) as well as for passive transportation (public transport and roads) [87]. We conclude that the heterogeneity in exposure assessment associated with built environmental variables made the examination of the associations with T2DM risk/prevalence more difficult.

Finally, although higher exposure to residential noise was consistently associated with higher T2DM risk/ prevalence in individual studies, this was not confirmed in our meta-analysis, in contrast with an earlier metaanalysis [16]. This difference could be explained by the inclusion of only confounder adjusted risk ratios in our study.

A strength of this study is the comprehensive overview of the literature on the association between built environmental characteristics and T2DM risk/prevalence, in which we included worldwide evidence. We assessed study quality and took country income levels into account. However, certain limitations of this study need to be addressed.

A weakness of any systematic review and metaanalysis is that its quality is dependent on the quality of the studies included. For instance, not all studies that were included distinguished between T2DM and type 1 diabetes mellitus. However, the majority of all people with diabetes have T2DM so the evidence provided in our review was very likely applicable to T2DM risk/ prevalence [1]. Secondly, because most studies in the present review were cross-sectional, our review cannot provide the foundation for causal inferences. Finally, publication bias could influence our findings, but our search turned out a relatively high number of null findings, suggesting publication bias an unlikely limitation. Finally, residential self-selection is an important issue that should be included in studies investigating the associations between built environment and disease. Selfselection occurs when residents choose a residence based on socioeconomic or other circumstances, or lifestyle preferences. Evidently, such selections may influence our results, as for instance higher socioeconomic status neighbourhoods may contain more green space, as well as more highly educated and health-conscious residents. However, the true effect of residential selfselection on these associations has often not been accounted for in the included studies and is difficult to investigate. One narrative review observed that studies using various approaches to identify self-selection (i.e. a questionnaire or statistical methods) explained only a minor part of the associations between built environment and travel behaviours [88]. Two studies included in the present review observed that residential relocation, as an indicator of self-selection, resulted in inconsistent effects on associations with health outcomes [57, 58]. It is, therefore, hard to conclude on the effect of self-selection bias on our results, based on the current evidence.

Despite the limitations of our study, our results may be relevant for infrastructure planning. For example, in addition to other positive consequences of walkability and access to green space, these environmental characteristics may also contribute to T2DM prevention. Future research should focus on developing a more homogeneous definition of environmental characteristics, particularly in relation to the food environment. Also, more in-depth explorations are necessary of the pathways through which environments affect diabetes risk, while taking the potential confounding variables into account.

\section{Conclusions}

In conclusion, urbanisation is associated with higher T2DM risk/prevalence. The built physical activity environment - walkability and access to green space, in particular - was consistently associated with reduced T2DM risk/prevalence, while no consistent evidence was found for an association between the built food environment and T2DM risk/prevalence. These conclusions have implications in terms of urban planning and the inclusion of walkable and green cities.

\section{Additional files}

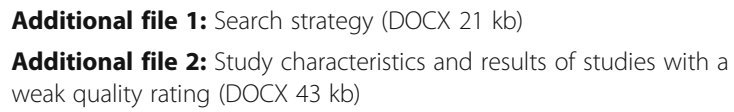

Additional file 3: Sensitivity analyses (ZIP $120 \mathrm{~kb}$ )

Additional file 4: Study characteristics and results of studies investigating combination environmental characteristics. (DOCX $21 \mathrm{~kb}$ )

Funding

The authors declare that this research received no funding. As a corresponding author, I confirm that I had full access to all data and the final responsibility for the decision to submit for publication. 


\section{Availability of data and material}

The data sets used and/or analysed during the current study are available from the corresponding author on reasonable request.

\section{Authors' contributions}

$\mathrm{NdB}$ performed the literature search, study selection, data extraction, quality assessment and data synthesis, and drafted the manuscript, tables and figures. JL performed study selection and quality assessment, assessed data extraction and made major revisions to the manuscript. FR provided support in design and execution of the review and meta-analyses, and made major revisions to the manuscript. $\downarrow J$ performed the literature search and contributed to drafting the methods section and flow chart of inclusion. JB provided support in design and execution of the review and meta-analyses, and made major revisions to the manuscript. JWJB performed study selection and quality assessment, made major revisions to the manuscript, is the guarantor of this work and takes responsibility for the integrity of the work and analyses. This manuscript has not been submitted elsewhere and it is original.

\section{Competing interests}

The authors declare that they have no competing interests.

\section{Publisher's Note}

Springer Nature remains neutral with regard to jurisdictional claims in published maps and institutional affiliations.

\section{Author details}

'Department of Epidemiology \& Biostatistics, Amsterdam Public Health Research Institute, VU University Medical Center, De Boelelaan 1089a, 1081 HV Amsterdam, The Netherlands. ${ }^{2}$ University Library, VU, Amsterdam, The Netherlands. ${ }^{3}$ Amsterdam School for Communication Research, University of Amsterdam, Amsterdam, The Netherlands. ${ }^{4}$ Julius Center for Health Sciences and Primary Care, University Medical Center Utrecht, Utrecht, The Netherlands.

Received: 31 July 2017 Accepted: 15 December 2017

Published online: 31 January 2018

\section{References}

1. WHO. Diabetes, Fact Sheet. WHO; 2016. http://www.who.int/mediacentre/ factsheets/fs312/en/. Accessed Sept 2016.

2. Cappuccio FP, D'Elia L, Strazzullo P, Miller MA. Quantity and quality of sleep and incidence of type 2 diabetes: a systematic review and meta-analysis. Diabetes Care. 2010:33(2):414-20.

3. Teixeira PJ, Carraca EV, Marques MM, Rutter H, Oppert JM, De Bourdeaudhuij I, et al. Successful behavior change in obesity interventions in adults: a systematic review of self-regulation mediators. BMC Med. 2015;13:84.

4. Van Holle V, Deforche B, Van Cauwenberg J, Goubert L, Maes L, Van de Weghe $N$, et al. Relationship between the physical environment and different domains of physical activity in European adults: a systematic review. BMC Public Health. 2012;12:807.

5. Sallis JF, Cerin E, Conway TL, Adams MA, Frank LD, Pratt M, et al. Physical activity in relation to urban environments in 14 cities worldwide: a crosssectional study. Lancet. 2016;387(10034):2207-17.

6. Osei-Kwasi HA, Nicolaou M, Powell K, Terragni L, Maes L, Stronks K, et al. Systematic mapping review of the factors influencing dietary behaviour in ethnic minority groups living in Europe: a DEDIPAC study. Int J Behav Nutr Phys Act. 2016;13:85.

7. Popkin BM. Nutrition transition and the global diabetes epidemic. Curr Diab Rep. 2015;15(9):64

8. Cho N, Whiting D, et al. Diabetes Atlas, 7th ed. International Diabetes Federation; 2015. http://www.oedg.at/pdf/1606_IDF_Atlas_2015_UK.pdf.

9. Caspi CE, Sorensen G, Subramanian S, Kawachi I. The local food environment and diet: a systematic review. Health Place. 2012;18(5):1172-87.

10. Fraser LK, Edwards KL, Cade J, Clarke GP. The geography of fast food outlets: a review. Int J Environ Res Public Health. 2010;7(5):2290-308.

11. Ising $H$, Braun $C$. Acute and chronic endocrine effects of noise: review of the research conducted at the Institute for Water. Soil Air Hygiene Noise Health. 2000;2(7):7-24.

12. Pirrera S, De Valck E, Cluydts R. Nocturnal road traffic noise: a review on its assessment and consequences on sleep and health. Environ Int. 2010;36(5):492-8.
13. Angkurawaranon C, Jiraporncharoen W, Chenthanakij B, Doyle P, Nitsch D. Urbanization and non-communicable disease in Southeast Asia: a review of current evidence. Public health. 2014;128(10):886-95.

14. Schulz M, Romppel M, Grande G. Built environment and health: a systematic review of studies in Germany. J Public Health. 2016. https://doi.org/10.1093/ pubmed/fdw141.

15. Malambo P, Kengne AP, De Villiers A, Lambert EV, Puoane T. Built environment, selected risk factors and major cardiovascular disease outcomes: a systematic review. PLoS One. 2016;11(11):e0166846.

16. Dzhambov AM. Long-term noise exposure and the risk for type 2 diabetes: a meta-analysis: erratum. Noise Health. 2015;17(75):123.

17. Mackenbach JD, Rutter H, Compernolle S, Glonti K, Oppert JM, Charreire H, et al. Obesogenic environments: a systematic review of the association between the physical environment and adult weight status, the SPOTLIGHT project. BMC Public Health. 2014;14:233.

18. World Bank list of economies, 2016. http://www.ispo2017.org/wp-content/ uploads/2016/11/World-Bank-List-of-Economies.pdf. Accessed Sept 2016.

19. Booth GL, Creatore MI, Moineddin R, Gozdyra P, Weyman JT, Matheson Fl, et al. Unwalkable neighborhoods, poverty, and the risk of diabetes among recent immigrants to Canada compared with long-term residents. Diabetes Care. 2013; 36:302-5.

20. Creatore Ml, Glazier RH, Moineddin R, Fazli GS, Johns A, Gozdyra P, et al. Association of neighborhood walkability with change in overweight, obesity, and diabetes. JAMA. 2016;315(20):2211-20.

21. Glazier RH, Creatore MI, Weyman JT, Fazli G, Matheson FI, Gozdyra P, et al. Density, destinations or both? A comparison of measures of walkability in relation to transportation behaviors, obesity and diabetes in Toronto, Canad. PLoS One. 2014;9(1):e85295.

22. Sundquist K, Eriksson U, Mezuk B, Ohlsson H. Neighborhood walkability, deprivation and incidence of type 2 diabetes: a population-based study on 512,061 Swedish adults. Health Place. 2015;31:24-30.

23. Cubbin C, Sundquist K, Ahlen H, Johansson SE, Winkleby MA, Sundquist J. Neighborhood deprivation and cardiovascular disease risk factors: protective and harmful effects. Scand J Public Health. 2006;34(3):228-37.

24. Danesh J, Collins R, Appleby P, Peto R. Association of fibrinogen, C-reactive protein, albumin, or leukocyte count with coronary heart disease: metaanalyses of prospective studies. JAMA. 1998;279(18):1477-82.

25. Dar HI, Dar SH, Bhat RA, Kamili MA, Mir SR. Prevalence of type 2 diabetes mellitus and its risk factors in the age group 40 years and above in the Kashmir valley of the Indian subcontinent. JIACM. 2015;16(3-4):187-97.

26. Mi T, Sun S, Du Y, Guo S, Cong L, Cao M, et al. Differences in the distribution of risk factors for stroke among the high-risk population in urban and rural areas of Eastern China. Brain Behav. 2016;6(5): e00461.

27. Kodaman N, Aldrich MC, Sobota R, Asselbergs FW, Poku KA, Brown NJ, et al. Cardiovascular disease risk factors in Ghana during the rural-to-urban transition: a cross-sectional study. PLoS One. 2016:11(10):e0162753.

28. Gangquiang D, Ming Y, Weiwei G, Ruying H, MacLennan R. Nutrition-related disease and death in Zhejiang Province. Asia Pacific J Clin Nutr. 2004;13(2):162-5.

29. Azizi F, Vazirian P, Dolatshi P, Habibian S. Screening for type 2 diabetes in the Iranian national programme: a preliminary report. East Mediterr Health J. 2003; 9(5-6):1122-7.

30. Mierzecki A, Kloda K, Gryko A, Czarnowski D, Chelstowski K, Chlabicz S. Atherosclerosis risk factors in rural and urban adult populations living in Poland. Exp Clin Cardiol. 2014;20(8):3152-7.

31. Njelekela M, Sato T, Nara Y, Miki T, Kuga S, Noguchi T, et al. Nutritional variation and cardiovascular risk factors in Tanzania - rural-urban difference. S Afr Med J. 2003;93(4):295-9.

32. Ceesay MM, Morgan MW, Kamanda MO, Willoughby VR, Lisk DR. Prevalence of diabetes in rural and urban populations in southern Sierra Leone: a preliminary survey. Trop Med Int Health. 1997;2(3):272-7.

33. Asadollahi K, Delpisheh A, Asadollahi P, Abangah G. Hyperglycaemia and its related risk factors in llam province, west of Iran - a population-based study. J Diabetes Metab Disord. 2015;14:81.

34. Bharati D, Pal R, Rekha R, Yamuna T, Kar S, Radjou A. Ageing in Puducherry, South India: an overview of morbidity profile. J Pharm Bioallied Sci. 2011;3(4):537-42.

35. Colleran KM, Richards A, Shafer K. Disparities in cardiovascular disease risk and treatment: demographic comparison. J Investig Med. 2007:55(8):415-22.

36. Khan MM, Gruebner $O$, Kraemer A. The geography of diabetes among the general adults aged 35 years and older in Bangladesh: recent evidence from a cross-sectional survey. PLoS One. 2014;9(10):e110756. 
37. Nakibuuka J, Sajatovic M, Nankabirwa J, Furlan AJ, Kayima J, Ddumba E, et al. Stroke-risk factors differ between rural and urban communities: population survey in Central Uganda. Neuroepidemiology. 2015;44(3):156-65.

38. Shera AS, Jawad F, Maqsood A. Prevalence of diabetes in Pakistan. Diabetes Res Clin Pract. 2007;76(2):219-22.

39. Valverde JC, Tormo MJ, Navarro C, Rodriguez-Barranco M, Marco R, Egea JM, et al. Prevalence of diabetes in Murcia (Spain): a Mediterranean area characterised by obesity. Diabetes Res Clin Pract. 2006;71(2):202-9.

40. Mohamud WN, Ismail AA, Sharifuddin A, Ismail IS, Musa KI, Kadir KA, et al. Prevalence of metabolic syndrome and its risk factors in adult Malaysians: results of a nationwide survey. Diabetes Res Clin Pract. 2011;91(2):239-45.

41. Bahendeka S, Wesonga R, Mutungi G, Muwonge J, Neema S, Guwatudde D. Prevalence and correlates of diabetes mellitus in Uganda: a populationbased national survey. Trop Med Int Health. 2016;21(3):405-16.

42. Astell-Burt T, Feng X, Kolt GS. Is neighborhood green space associated with a lower risk of type 2 diabetes? Evidence from 267,072 Australians. Diabetes Care. 2014;37(1):197-201

43. Shaffer K, Bopp M, Papalia Z, Sims D, Bopp CM. The relationship of living environment with behavioral and fitness outcomes by sex: an exploratory study in college-aged students. Int J Exerc Sci. 2017;10(3):330-9.

44. Bodicoat DH, O'Donovan G, Dalton AM, Gray LJ, Yates T, Edwardson C, et al. The association between neighbourhood greenspace and type 2 diabetes in a large cross-sectional study. BMJ Open. 2014;4(12):e006076.

45. Lee H, Kang HM, Ko YJ, Kim HS, Kim YJ, Bae WK, et al. Influence of urban neighbourhood environment on physical activity and obesity-related diseases. Public Health. 2015;129(9):1204-10.

46. Ahern M, Brown C, Dukas S. A national study of the association between food environments and county-level health outcomes. J Rural Health. 2011; 27(4):367-79.

47. Auchincloss AH, Diez Roux AV, Mujahid MS, Mingwu Shen MS, Bertoni AG, Carnethon MR. Neighborhood resources for physical activity and healthy foods and incidence of type 2 diabetes mellitus. Arch Intern Med. 2009; 169(18):1698-704.

48. Christine PJ, Auchincloss AH, Bertoni AG, Carnethon MR, Sanchez BN, Moore $\mathrm{K}$, et al. Longitudinal associations between neighborhood physical and social environments and incident type 2 diabetes mellitus: the multi-ethnic study of atherosclerosis (MESA). JAMA Intern Med. 2015;175(8):1311-20.

49. Cunningham-Myrie CA, Theall KP, Younger NO, Mabile EA, Tulloch-Reid MK, Francis DK, et al. Associations between neighborhood effects and physical activity, obesity, and diabetes: The Jamaica Health and Lifestyle Survey 2008. J Clin Epidemiol. 2015;68(9):970-8.

50. Eichinger M, Titze S, Haditsch B, Dorner TE, Stronegger WJ. How are physical activity behaviors and cardiovascular risk factors associated with characteristics of the built and social residential environment? PLoS One. 2015;10(6):e0126010

51. Herrick CJ, Yount BW, Eyler AA. Implications of supermarket access, neighbourhood walkability and poverty rates for diabetes risk in an employee population. Public Health Nutr. 2016;19(11):2040-8.

52. Marshall WE, Piatkowski DP, Garrick NW. Community design, street networks, and public health. J Transport Health. 2014;1(4):326-40.

53. Mena C, Fuentes E, Ormazabal Y, Palomo-Velez G, Palomo I. Role of access to parks and markets with anthropometric measurements, biological markers, and a healthy lifestyle. Int J Environ Health Res. 2015;25(4):373-83.

54. Paquet C, Coffee NT, Haren MT, Howard NJ, Adams RJ, Taylor AW, et al. Food environment, walkability, and public open spaces are associated with incident development of cardio-metabolic risk factors in a biomedical cohort. Health Place. 2014;28:173-6.

55. Salois MJ. Obesity and diabetes, the built environment, and the 'local' food economy in the United States, 2007. Econ Hum Biol. 2012;10(1):35-42.

56. Schootman M, Andresen EM, Wolinsky FD, Malmstrom TK, Miller JP, Yan Y, et al. The effect of adverse housing and neighborhood conditions on the development of diabetes mellitus among middle-aged African Americans. Am J Epidemiol. 2007;166(4):379-87.

57. Braun LM, Rodriguez DA, Evenson KR, Hirsch JA, Moore KA, Diez Roux AV. Walkability and cardiometabolic risk factors: cross-sectional and longitudinal associations from the multi-ethnic study of atherosclerosis. Health Place. 2016;39:9-17.

58. Braun LM, Rodriguez DA, Song Y, Meyer KA, Lewis CE, Reis JP, et al. Changes in walking, body mass index, and cardiometabolic risk factors following residential relocation: longitudinal results from the CARDIA study. J Transp Health. 2016;3(4):426-39.
59. Dalton AM, Jones AP, Sharp SJ, Cooper AJ, Griffin S, Wareham NJ. Residential neighbourhood greenspace is associated with reduced risk of incident diabetes in older people: a prospective cohort study. BMC Public Health. 2016;16(1):1171.

60. Fujiwara T, Takamoto I, Amemiya A, Hanazato M, Suzuki N, Nagamine Y, et al. Is a hilly neighborhood environment associated with diabetes mellitus among older people? Results from the JAGES 2010 study. Soc Sci Med. 2017;182:45-51.

61. Gebreab SY, Hickson DA, Sims M, Wyatt SB, Davis SK, Correa A, et al. Neighborhood social and physical environments and type 2 diabetes mellitus in African Americans: the Jackson Heart study. Health Place. 2017;43:128-37.

62. Loo CK, Greiver M, Aliarzadeh B, Lewis D. Association between neighbourhood walkability and metabolic risk factors influenced by physical activity: a cross-sectional study of adults in Toronto, Canada. BMJ Open. 2017;7(4):e013889.

63. Myers CA, Slack T, Broyles ST, Heymsfield SB, Church TS, Martin CK. Diabetes prevalence is associated with different community factors in the diabetes belt versus the rest of the United States. Obesity. 2017;25(2):452-9.

64. Ngom R, Gosselin P, Blais C, Rochette L. Type and proximity of green spaces are important for preventing cardiovascular morbidity and diabetes-a cross-sectional study for Quebec, Canada. Int J Environ Res Public Health. 2016;13(4):423.

65. Muller-Riemenschneider F, Pereira G, Villanueva K, Christian H, Knuiman M, Giles-Corti B, et al. Neighborhood walkability and cardiometabolic risk factors in Australian adults: an observational study. BMC Public Health. 2013;13:755.

66. Maas J, Verheij RA, de Vries S, Spreeuwenberg P, Schellevis FG, Groenewegen PP. Morbidity is related to a green living environment. J Epidemiol Community Health. 2009;63(12):967-73.

67. Attard SM, Herring AH, Mayer-Davis EJ, Popkin BM, Meigs JB, Gordon-Larsen P. Multilevel examination of diabetes in modernising China: what elements of urbanisation are most associated with diabetes? Diabetologia. 2012; 55(12):3182-92.

68. Freedman VA, Grafova IB, Rogowski J. Neighborhoods and chronic disease onset in later life. Am J Public Health. 2011;101(1):79-86.

69. AlHasan DM, Eberth JM. An ecological analysis of food outlet density and prevalence of type II diabetes in South Carolina counties. BMC Public Health. 2016;16:10

70. Mezuk B, Li X, Cederin K, Rice K, Sundquist J, Sundquist K. Beyond access: characteristics of the food environment and risk of diabetes. Am J Epidemiol. 2016;183(12):1129-37.

71. Carroll SJ, Paquet C, Howard NJ, Coffee NT, Adams RJ, Taylor AW Niyonsenga T, et al. Local descriptive body weight and dietary norms, food availability, and 10-year change in glycosylated haemoglobin in an Australian population-based biomedical cohort. BMC Public Health. 2017; 17(1):149.

72. Bodicoat DH, Carter P, Comber A, Edwardson C, Gray LJ, Hill S, et al. Is the number of fast-food outlets in the neighbourhood related to screendetected type 2 diabetes mellitus and associated risk factors? Public Health Nutr. 2015;18(9):1698-705.

73. Flynt A, Daepp MI. Diet-related chronic disease in the northeastern United States: a model-based clustering approach. Int J Health Geogr. 2015;14:25.

74. Frankenfeld CL, Leslie TF, Makara MA. Diabetes, obesity, and recommended fruit and vegetable consumption in relation to food environment sub-types: a cross-sectional analysis of Behavioral Risk Factor Surveillance System, United States Census, and food establishment data. BMC Public Health. 2015;15:491.

75. Morland K, Diez Roux AV, Wing S. Supermarkets, other food stores, and obesity: the atherosclerosis risk in communities study. Am J Prev Med. 2006; 30(4):333-9.

76. Babey SH, Diamant AL, Hastert TA, Harvey S. Designed for disease: the link between local food environments and obesity and diabetes. California Center for Public Health Advocacy, PolicyLink, and the UCLA Center for Health Policy Res. 2008.

77. Jiao J, Moudon AV, Kim SY, Hurvitz PM, Drewnowski A. Health implications of adults' eating at and living near fast food or quick service restaurants. Nutr Diabetes. 2015;5:e171.

78. Hipp JA, Chalise N. Spatial analysis and correlates of county-level diabetes prevalence, 2009-2010. Prev Chronic Dis. 2015;12:E08.

79. Liu L, Nunez AE. Multilevel and urban health modeling of risk factors for diabetes mellitus: a new insight into public health and preventive medicine. Adv Prev Med. 2014;2014:246049.

80. Braun LM, Malizia E. Downtown vibrancy influences public health and safety outcomes in urban counties. J Transport Health. 2015;2(4):540-8. 
81. Meyer KA, Boone-Heinonen J, Duffey KJ, Rodriguez DA, Kiefe Cl, Lewis CE, et al. Combined measure of neighborhood food and physical activity environments and weight-related outcomes: the CARDIA study. Health Place. 2015;33:9-18.

82. Cai $Y$, Hansell AL, Blangiardo M, Burton PR, BioShaRe, De Hoogh $K$, et al. Long-term exposure to road traffic noise, ambient air pollution, and cardiovascular risk factors in the HUNT and lifelines cohorts. Eur Heart J. 2017;38(29):2290-6.

83. Dzhambov A, Dimitrova D. Exposures to road traffic, noise, and air pollution as risk factors for type 2 diabetes: a feasibility study in Bulgaria. Noise Health. 2016;18(82):133-42.

84. Sorensen M, Andersen ZJ, Nordsborg RB, Becker T, Tjonneland A, Overvad K, et al. Long-term exposure to road traffic noise and incident diabetes: a cohort study. Environ Health Perspect. 2013;121(2):217-22.

85. Eriksson C, Hilding A, Pyko A, Bluhm G, Pershagen G, Ostenson CG. Long-term aircraft noise exposure and body mass index, waist circumference, and type 2 diabetes: a prospective study. Environ Health Perspect. 2014;122(7):687-94.

86. Heidemann C, Niemann H, Paprott R, Du Y, Rathmann W, Scheidt-Nave C. Residential traffic and incidence of type 2 diabetes: the German health interview and examination surveys. Diabet Med. 2014;31(10):1269-76.

87. Sallis JF, Cervero RB, Ascher W, Henderson KA, Kraft MK, Kerr J. An ecological approach to creating active living communities. Annu Rev Public Health. 2006;27:297-322.

88. Cao X, Mokhtarian PL, Handy SL. Examining the impacts of residential selfselection on travel behaviour: a focus on empirical findings. Transp Rev. 2009;29(3):359-95.

89. Aekplakorn W, Chariyalertsak $S$, Kessomboon $P$, Sangthong $R$, Inthawong $R$, Putwatana $P$, et al. Prevalence and management of diabetes and metabolic risk factors in Thai adults. Diabetes Care. 2011;34(9):1980-5.

90. Agyemang C, Meeks K, Beune E, Owusu-Dabo E, Mockenhaupt FP, Addo J, et al. Obesity and type 2 diabetes in sub-Saharan Africans - is the burden in today's Africa similar to African migrants in Europe? The RODAM study. BMC Med. 2016;14(1):166.

91. Ali O, Tan TT, Sakinah O, Khalid BAK, Wu LL, Ng ML. Prevalence of NIDDM and impaired glucose tolerance in aborigines and Malays in Malaysia and their relationship to sociodemographic, health, and nutritional factors. Diabetes Care. 1993;16(1):68-75.

92. Al-Moosa S, Allin S, Jemiai N, Al-Lawati J, Mossialos E. Diabetes and urbanization in the Omani population: an analysis of national survey data. Popul Health Metr. 2006;4:5.

93. Anjana RM, Pradeepa R, Deepa M, Datta M, Sudha V, Unnikrishnan R, et al. Prevalence of diabetes and prediabetes (impaired fasting glucose and/or impaired glucose tolerance) in urban and rural India: phase I results of the Indian Council of Medical Research-INdia DIABetes (ICMR-INDIAB) study. Diabetologia. 2011;54(12):3022-7.

94. Assah FK, Ekelund U, Brage S, Mbanya JC, Wareham NJ. Urbanization, physical activity, and metabolic health in sub-Saharan Africa. Diabetes Care. 2011;34(2):491-6.

95. Allender S, Wickramasinghe K, Goldacre M, Matthews D, Katulanda P. Quantifying urbanization as a risk factor for noncommunicable disease. J Urban Health. 2011;88(5):906-18.

96. Balde NM, Diallo I, Balde MD, Barry IS, Kaba L, Diallo MM, et al. Diabetes and impaired fasting glucose in rural and urban populations in Futa Jallon (Guinea): prevalence and associated risk factors. Diabetes Metab. 2007;33(2):114-20.

97. Balogun WO, Gureje O. Self-reported incident type 2 diabetes in the Ibadan study of ageing: relationship with urban residence and socioeconomic status. Gerontology. 2013;59(1):3-7.

98. Baltazar JC, Ancheta CA, Aban IB, Fernando RE, Baquilod MM. Prevalence and correlates of diabetes mellitus and impaired glucose tolerance among adults in Luzon, Philippines. Diabetes Res Clin Pract. 2004;64(2):107-15.

99. Bernabe-Ortiz A, Carrillo-Larco RM, Gilman RH, Miele CH, Checkley W, Wells $J$, et al. Geographical variation in the progression of type 2 diabetes in Peru: the CRONICAS cohort study. Diabetes Res Clin Pract. 2016;121:135-45.

100. Bocquier A, Cortaredona S, Nauleau S, Jardin M, Verger P. Prevalence of treated diabetes: geographical variations at the small-area level and their association with area-level characteristics. A multilevel analysis in Southeastern France. Diabetes Metab. 2011;37(1):39-46.

101. Christensen DL, Friis H, Mwaniki DL, Kilonzo B, Tetens I, Boit MK, et al. Prevalence of glucose intolerance and associated risk factors in rural and urban populations of different ethnic groups in Kenya. Diabetes Res Clin Pract. 2009;84(3):303-10.
102. Dagenais GR, Gerstein HC, Zhang X, McQueen M, Lear S, Lopez-Jaramillo P, et al. Variations in diabetes prevalence in low-, middle-, and high-income countries: results from the prospective urban and rural epidemiological study. Diabetes Care. 2016;39(5):780-7.

103. Davila EP, Quintero MA, Orrego ML, Ford ES, Walke H, Arenas MM, et al. Prevalence and risk factors for metabolic syndrome in Medellin and surrounding municipalities, Colombia, 2008-2010. Prev Med. 2013;56(1):30-4.

104. Delisle H, Ntandou-Bouzitou G, Agueh V, Sodjinou R, Fayomi B. Urbanisation, nutrition transition and cardiometabolic risk: the Benin study. Br J Nutr. 2012;107(10):1534-44.

105. Dong Y, Gao W, Nan H, Yu H, Li F, Duan W, et al. Prevalence of type 2 diabetes in urban and rural Chinese populations in Qingdao, China. Diabet Med. 2005;22(10):1427-33.

106. Du GL, Su YX, Yao H, Zhu J, Ma Q, Tuerdi A, et al. Metabolic risk factors of type 2 diabetes mellitus and correlated glycemic control/complications: a cross-sectional study between rural and urban Uygur residents in Xinjiang Uygur Autonomous Region. PLoS One. 2016;11(9):e0162611.

107. Esteghamati A, Meysamie A, Khalilzadeh O, Rashidi A, Haghazali M, Asgari F, et al. Third national surveillance of risk factors of non-communicable diseases (SURFNCD-2007) in Iran: methods and results on prevalence of diabetes, hypertension, obesity, central obesity, and dyslipidemia. BMC Public Health. 2009;9:167.

108. Georgousopoulou EN, Mellor DD, Naumovski N, Polychronopoulos E, Tyrovolas S, Piscopo S, et al. Mediterranean lifestyle and cardiovascular disease prevention. Cardiovasc Diagn Ther. 2017;7 Suppl 1:S39-47.

109. Gong H, Pa L, Wang K, Mu H, Dong F, Ya S, et al. Prevalence of diabetes and associated factors in the Uyghur and Han population in Xinjiang, China. Int J Environ Res Public Health. 2015;12(10):12792-802.

110. Hussain A, Rahim MA, Azad Khan AK, Ali SMK, Vaaler S. Type 2 diabetes in rural and urban population: diverse prevalence and associated risk factors in Bangladesh. Diabet Med. 2005;22(7):931-6.

111. Han SJ, Kim HJ, Kim DJ, Lee KW, Cho NH. Incidence and predictors of type 2 diabetes among Koreans: a 12-year follow up of the Korean genome and epidemiology study. Diabetes Res Clin Pract. 2017;123:173-80.

112. Katchunga P, Masumbuko B, Belma M, Kashongwe Munogolo Z, Hermans MP, M'Buyamba-Kabangu JR. Age and living in an urban environment are major determinants of diabetes among South Kivu Congolese adults. Diabetes Metab. 2012;38(4):324-31.

113. Keel S, Foreman J, Xie J, van Wijngaarden P, Taylor HR, Dirani M. The prevalence of self-reported diabetes in the Australian national eye health survey. PLoS One. 2017;12(1):e0169211.

114. Mayega RW, Guwatudde D, Makumbi F, Nakwagala FN, Peterson S, Tomson G, et al. Diabetes and pre-diabetes among persons aged 35 to 60 years in eastern Uganda: prevalence and associated factors. PLoS One. 2013;8(8):e72554.

115. Mohan I, Gupta R, Misra A, Sharma KK, Agrawal A, Vikram NK, et al. Disparities in prevalence of cardiometablic risk factors in rural, urban-poor, and urban-middle class women in India. PLoS One. 2016;11(2):e0149437.

116. Msyamboza KP, Mvula CJ, Kathyola D. Prevalence and correlates of diabetes mellitus in Malawi: population-based national NCD STEPS survey. BMC Endocr Disord. 2014;14(1):41.

117. Ntandou G, Delisle H, Agueh V, Fayomi B. Abdominal obesity explains the positive rural-urban gradient in the prevalence of the metabolic syndrome in Benin. West Africa Nutr Res. 2009;29(3):180-9.

118. Oyebode O, Pape UJ, Laverty AA, Lee JT, Bhan N, Millett C. Rural, urban and migrant differences in non-communicable disease risk-factors in middle income countries: a cross-sectional study of WHO-SAGE data. PLoS One. 2015;10(4):e0122747.

119. Papoz L, Bamy S, Simon D, Group CS. Prevalence of diabetes mellitus in New Caledonia: ethnic and urban-rural differences. Am J Epidemiol. 1996; 143(10):1018-24.

120. Pham NM, Eggleston K. Prevalence and determinants of diabetes and prediabetes among Vietnamese adults. Diabetes Res Clin Pract. 2016; 113:116-24.

121. Raghupathy P, Antonisamy B, Fall CH, Geethanjali FS, Leary SD, Saperia J, et al. High prevalence of glucose intolerance even among young adults in south India. Diabetes Res Clin Pract. 2007;77(2):269-79.

122. Ramdani N, Vanderpas J, Boutayeb A, Meziane A, Hassani B, Zoheir J, et al. Diabetes and obesity in the eastern Morocco. Mediterr J Nutr Metab. 2011; 5(2):149-55

123. Sadikot SM, Nigam A, Das S, Bajaj S, Zargar AH, Prasannakumar KM, et al. The burden of diabetes and impaired fasting glucose in India using the 
ADA 1997 criteria: prevalence of diabetes in India study (PODIS). Diabetes Res Clin Pract. 2004;66(3):293-300

124. Sobngwi E, Mbanya JC, Unwin NC, Porcher R, Kengne AP, Fezeu L, et al. Exposure over the life course to an urban environment and its relation with obesity, diabetes, and hypertension in rural and urban Cameroon. Int J Epidemiol. 2004;33(4):769-76.

125. Stanifer JW, Egger JR, Turner EL, Thielman N, Patel UD. Neighborhood clustering of non-communicable diseases: results from a community-based study in Northern Tanzania. BMC Public Health. 2016;16:226.

126. Weng X, Liu Y, Ma J, Wang W, Yang G, Caballero B. An urban-rural comparison of the prevalence of the metabolic syndrome in Eastern China. Public Health Nutr. 2007;10(2):131-6.

127. Wu J, Cheng X, Qiu L, Xu T, Zhu G, Han J, et al. Prevalence and clustering of major cardiovascular risk factors in China: a recent cross-sectional survey. Medicine. 2016:95(10):e2712.

128. Zhou M, Astell-Burt T, Bi Y, Feng X, Jiang Y, Li Y, et al. Geographical variation in diabetes prevalence and detection in China: multilevel spatial analysis of 98,058 adults. Diabetes Care. 2015;38(1):72-81.

Submit your next manuscript to BioMed Central and we will help you at every step:

- We accept pre-submission inquiries

- Our selector tool helps you to find the most relevant journal

- We provide round the clock customer support

- Convenient online submission

- Thorough peer review

- Inclusion in PubMed and all major indexing services

- Maximum visibility for your research

Submit your manuscript at www.biomedcentral.com/submit
Biomed Central 